\title{
Fibulin-1 regulates the pathogenesis of tissue remodeling in respiratory diseases
}

\author{
Gang Liu, ${ }^{1}$ Marion A. Cooley, ${ }^{2}$ Andrew G. Jarnicki, ${ }^{1}$ Alan C-Y. Hsu, ${ }^{1}$ Prema M. Nair, ${ }^{1}$ Tatt Jhong Haw, ${ }^{1}$ \\ Michael Fricker, ${ }^{1}$ Shaan L. Gellatly, ${ }^{1}$ Richard Y. Kim, ${ }^{1}$ Mark D. Inman, ${ }^{3}$ Gavin Tjin, ${ }^{4}$ Peter A.B. Wark, ${ }^{1,5}$ \\ Marjorie M. Walker, ${ }^{1}$ Jay C. Horvat, ${ }^{1}$ Brian C. Oliver, ${ }^{4,6}$ W. Scott Argraves, ${ }^{2}$ Darryl A. Knight, ${ }^{1,7}$ \\ Janette K. Burgess, ${ }^{4,8,9}$ and Philip M. Hansbro' ${ }^{1}$ \\ 'Priority Research for Healthy Lungs, Hunter Medical Research Institute and The University of Newcastle, Newcastle, \\ New South Wales, Australia. 'Department of Regenerative Medicine and Cell Biology, Medical University of South Carolina, \\ Charleston, South Carolina, USA. ${ }^{3}$ Division of Respirology, Department of Medicine, McMaster University, Hamilton, \\ Ontario, Canada. ${ }^{4}$ Woolcock Institute of Medical Research, Discipline of Pharmacology, The University of Sydney, Sydney, \\ New South Wales, Australia. ${ }^{5}$ Department of Respiratory and Sleep Medicine, John Hunter Hospital, Newcastle, \\ New South Wales, Australia. ${ }^{6}$ School of Life Sciences, The University of Technology, Sydney, New South Wales, Australia. \\ 7Department of Anesthesiology, Pharmacology and Therapeutics, The University of British Columbia, Vancouver, \\ British Columbia, Canada. ${ }^{8}$ Discipline of Pharmacology, Sydney Medical School, The University of Sydney, New South \\ Wales, Australia. ${ }^{9}$ Department of Pathology and Medical Biology, University of Groningen, University Medical Center, \\ Groningen, Netherlands.
}

\begin{abstract}
Airway and/or lung remodeling, involving exaggerated extracellular matrix (ECM) protein deposition, is a critical feature common to pulmonary diseases including chronic obstructive pulmonary disease (COPD), asthma, and idiopathic pulmonary fibrosis (IPF). Fibulin-1 (Fbln1), an important ECM protein involved in matrix organization, may be involved in the pathogenesis of these diseases. We found that Fbln1 was increased in COPD patients and in cigarette smokeinduced (CS-induced) experimental COPD in mice. Genetic or therapeutic inhibition of Fbln1c protected against CS-induced airway fibrosis and emphysema-like alveolar enlargement. In experimental COPD, this occurred through disrupted collagen organization and interactions with fibronectin, periostin, and tenascin-c. Genetic inhibition of Fbln1c also reduced levels of pulmonary inflammatory cells and proinflammatory cytokines/chemokines (TNF- $\alpha$, IL-33, and CXCL1) in experimental COPD. Fbln1c ${ }^{-/-}$mice also had reduced airway remodeling in experimental chronic asthma and pulmonary fibrosis. Our data show that Fbln1c may be a therapeutic target in chronic respiratory diseases.
\end{abstract}

Authorship note: G. Liu, M.A. Cooley, and A.G. Jarnicki contributed equally to this work. W. Scott Argraves is deceased.

Conflict of interest: The authors have declared that no conflict of interest exists.

Submitted: January 11, 2016 Accepted: May 11, 2016

Published: June 16, 2016

Reference information: JCI Insight. 2016;1(9):e86380. doi:10.1172/jci.insight.86380.

\section{Introduction}

The extracellular matrix (ECM) of the airways and lung is a dynamic structure composed of a diverse set of proteins, glycoproteins, and lipids that provides architectural support and contributes to biological processes in these tissues. Remodeling of the ECM occurs continuously and is tightly controlled to maintain homeostasis through the production of proteins including fibronectin ( $F n)$, fibulin (Fbln), periostin (Postn) and tenascin-c (Tnc), as well as their degradation by matrix (matrix metalloproteases; MMPs) and disintegrin and metalloproteinase with thrombospondin motifs (a disintegrin and metalloproteinase with thrombospondin motif; ADAMTS) metalloproteases (1). Disruption of homeostatic remodeling processes through changes in the levels and spatial and temporal production of ECM proteins leading to tissue remodeling is an important feature of chronic respiratory disease and other diseases.

Chronic obstructive pulmonary disease (COPD) is a progressive lung disease that is primarily caused by cigarette smoke-induced (CS-induced) chronic inflammation in Western societies (2-4). CS exposure also induces airway epithelial and mesenchymal cells to produce excessive amounts of ECM proteins (5). This also promotes COPD pathogenesis by causing airway remodeling and disrupting interstitial tissue and alveolar integrity. These events lead to airway narrowing, parenchymal damage, emphysema, and impaired lung function (6). Accumulation of ECM also occurs in specific regions of the lungs in asthma and 
idiopathic pulmonary fibrosis (IPF) $(7,8)$. Targeting ECM proteins may have beneficial effects; however, current therapies have limited efficacy in controlling tissue remodeling, and new therapeutic targets and strategies are urgently needed.

Fbln1 is a secreted glycoprotein (9) that stabilizes ECM integrity through interactions with other ECM proteins $(10,11)$. Four Fbln1 variants $(\mathrm{Fbln} 1 \mathrm{a} / \mathrm{b} / \mathrm{c} / \mathrm{d})$ have been identified with differences in C-terminal sequences. It is difficult to study the levels of different Fbln1 isoforms in human disease since antibodies to the specific isoforms are not available. Only Fbln1c and - $d$ are found in both humans and mice (12). Fbln1c, but not $-\mathrm{d}$, is implicated in airway remodeling and is specifically induced by TGF- $\beta$ treatment of airway smooth muscle (ASM) cells (13).

Fbln1 is known to play important roles in wound repair $(13,14)$ and is associated with several respiratory diseases. Increased levels of Fbln1 occur in serum and bronchoalveolar lavage fluid (BALF) of asthma patients, and inhibition of Fbln1c expression by antisense oligonucleotide reduced the proliferation of ASM cells from these patients (13). In IPF, ECM proteins are increased in both plasma and lung tissue (15), and Fbln1c variant-specific peptide increases the proliferation of lung fibroblasts, the main producers of ECM, in COPD and IPF patients (14). Cytokines associated with lung diseases, such as TGF- $\beta$, that are known to induce ECM proteins (16) also stimulate Fbln1 production in ASM cells from COPD patients, further indicating roles for Fbln1 in airway remodeling (17). Nevertheless, the in vivo function of Fbln1 in tissue remodeling in chronic pulmonary diseases is poorly understood.

In this study, we demonstrate that Fbln1 plays major roles in the pathogenesis of airway and lung remodeling in experimental COPD, asthma, and pulmonary fibrosis. Genetic or therapeutic inhibition of Fbln1c in experimental COPD prevented or reversed the development of CS-induced airway remodeling, emphysema-like alveolar enlargement, and inflammation, resulting in improved lung function. Targeting Fbln1 may therefore be beneficial in chronic respiratory disease and other diseases.

\section{Results}

Fbln1 is increased in primary bronchoepithelial cells ( $p B E C S$ ) from COPD patients. pBECs were obtained from COPD patients, non-COPD smokers, and nonsmoking healthy controls and were cultured. Proteins were extracted from cell lysates, and Fbln1 levels were measured by immunoblot. Fbln1 protein levels were significantly increased in the pBECs from COPD patients compared with both healthy controls and nonCOPD smokers (Figure 1A). Levels were also increased in the serum of COPD patients compared with healthy controls (Figure 1B).

Chronic CS exposure induces airway and lung remodeling in experimental COPD in mice. The effect of CS exposure on airway and lung remodeling was determined by assessing collagen levels in whole lungs and around the small airways in our well-established model of experimental COPD in C57BL/6 mice (18-22). In those and the current studies, we have shown extensively that 8 weeks of CS exposure resulted in the development of experimental COPD with airway and lung inflammation and remodeling, emphysema (increased alveolar diameter), and impaired lung function (as shown in subsequent Figures). These events were associated with reduced levels of total collagen in whole lungs compared with normal air-exposed mice determined by collagen-specific amino acid hydroxyproline analysis (Figure 1C). Soluble collagen levels were also significantly decreased. ECM gene array and quantitative PCR (qPCR) showed that the mRNA levels of type I collagen- $\alpha 1$ (Colla1), the most abundant collagen, and Col5a1 were lower in CS-exposed mice compared with normal air-exposed control mice (Supplemental Figure 1A and Supplemental Table 1; supplemental material available online with this article; doi:10.1172/jci. insight.86380DS1). These reductions are likely due to emphysema and loss of tissue. In contrast, collagen deposition around the small airways was significantly increased (Figure 1D).

Fbln1 protein levels in whole lungs and around small airways were significantly increased after 8 weeks of CS exposure but not at earlier timepoints (Figure 1, E and F). The histology showed that Fbln1c was produced in the airway epithelium after 6 weeks and then deposited around the airways after 8 weeks. This shows associations of increased Fbln1 concomitant with the development of disease features in experimental COPD, which replicated the increased levels of Fbln1 in the airways/lung tissues of COPD patients. However, Fbln1c and Fbln1d mRNA expression levels in lungs were not affected by experimental CS exposure (Supplemental Figure 1, B and C). Serum levels of Fbln1 protein were also increased in experimental COPD (Figure $1 \mathrm{G}$ ), as they were in human patients.

Generation of Fbln1 $1 c^{-1-}$ mice. Deletion of all variants of Fbln1 in mice results in perinatal lethality 
A
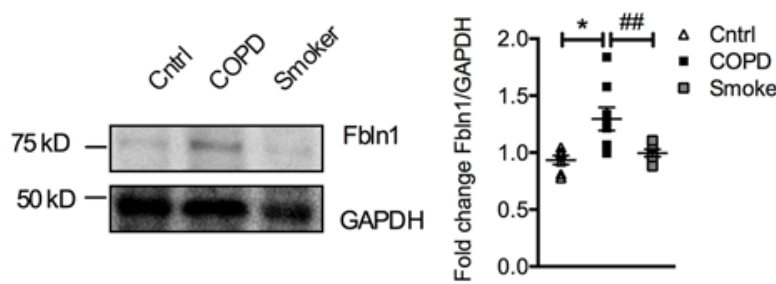

B

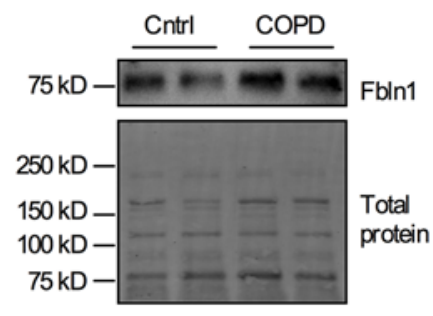

$\triangle$ Cntrl

- COPD

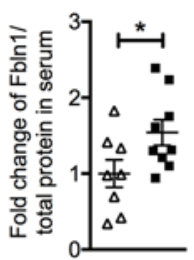

C
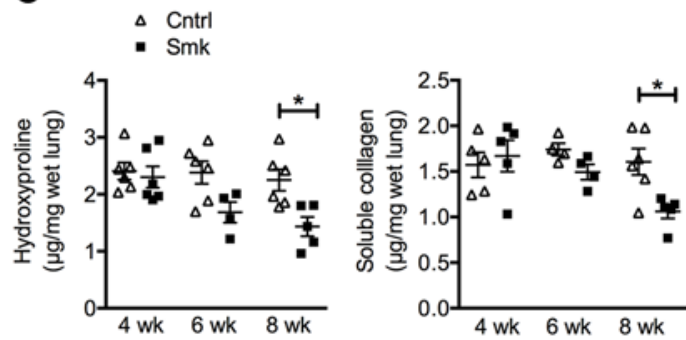

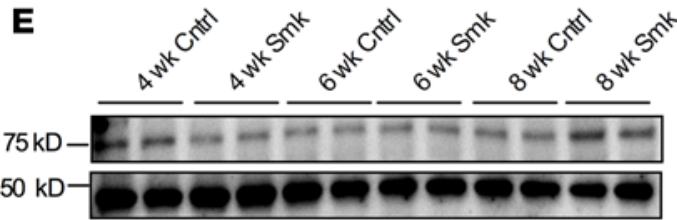

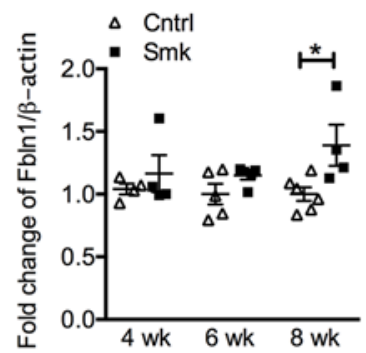

$\mathbf{F}$

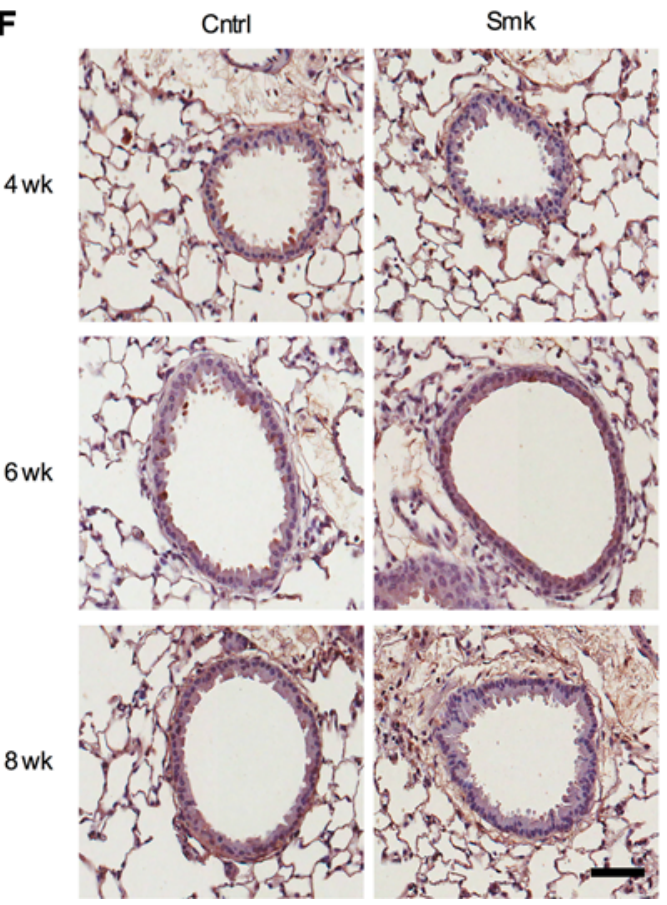

D
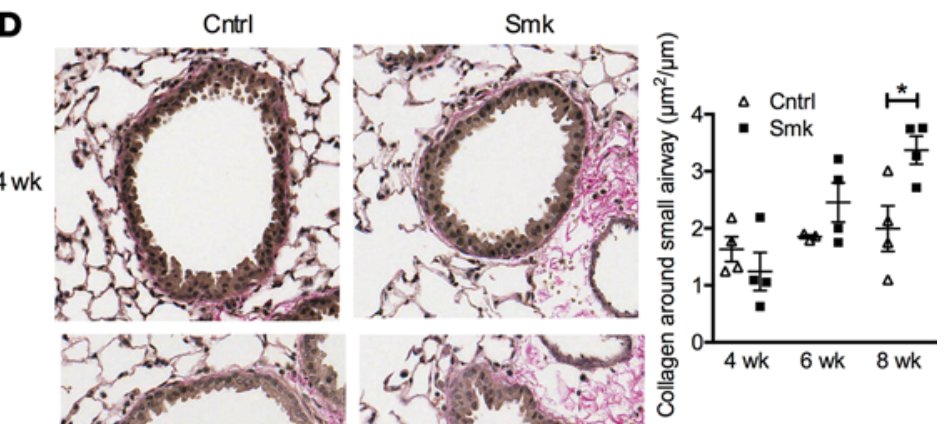

$6 \mathrm{wk}$

Fbln1

$\beta$-actin

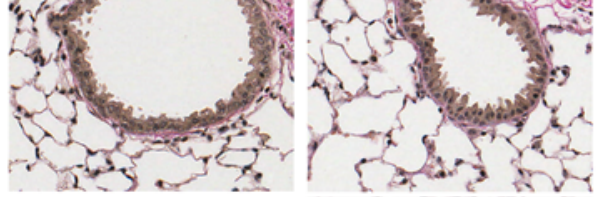

8 wk

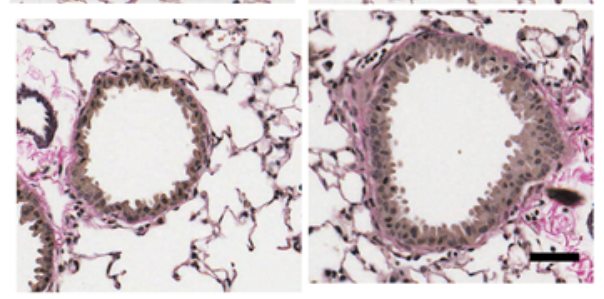

G

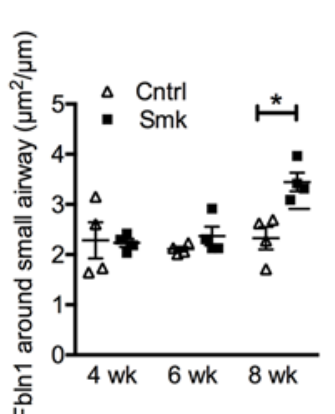

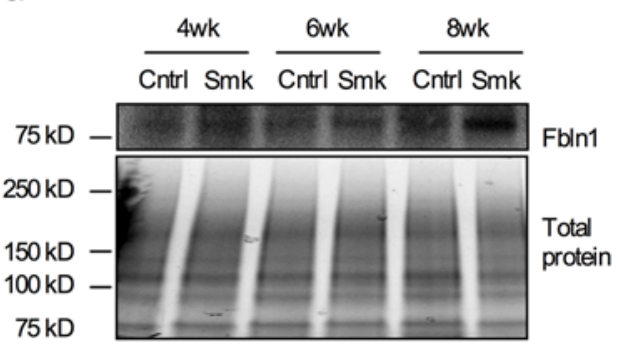

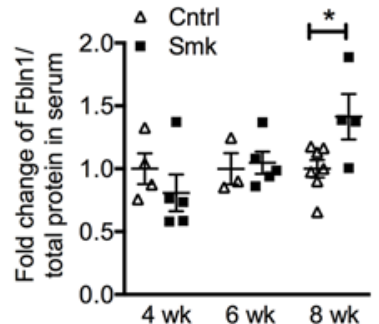


Figure 1. Fbln1 is increased in human COPD patients and cigarette smoke-induced (CS-induced) experimental COPD in mice. Primary bronchoepithelial cells ( $\mathrm{pBECs}$ ) and serum were collected from COPD patients, non-COPD smokers, and nonsmoking healthy controls. (A) Fbln1 protein in pBEC lysates assessed by immunoblot (left), and fold change of densitometry normalized to GAPDH (right). $n=5-9$. (B) Fbln1 protein in serum by immunoblot (left), and fold change of densitometry normalized to total protein (right). $n=8-9 .{ }^{*} P<0.05$ compared with human nonsmoking control, unpaired 2-tailed Student's $t$ test. WT mice were exposed to CS for 8 weeks to induce experimental COPD; controls were exposed to normal air. $n=5-6$. (C) Time course of total (left) and soluble collagen (right) in whole lungs. (D) Time course of collagen deposition around airways in lung sections stained with Verhoeff's-Van Gieson (VVG, left; scale bar: $50 \mu \mathrm{m}$ ) and quantified by normalization to perimeter of basement membrane (Pbm) (right). $n=24-40$ airways from $n=4-6$ mice per group. (E) Fbln1 protein in mouse lungs by immunoblot (top), and fold change of densitometry normalized to $\beta$-actin (bottom). $n=5$-6. (F) Time course of Fbln1 protein around small airways by IHC (left; scale bar: $50 \mu \mathrm{m}$ ), and quantification normalized to Pbm (right). $n=24-32$ airways from $n=4-6$ mice per group. (G) Time course of Fbln1 protein in serum, and fold change of densitometry normalized to total protein. $n=5-6$. Results are mean \pm SEM. ${ }^{*} P<0.05$ compared with human nonsmoking control or normal air-exposed WT mice controls. ${ }^{\#} P<0.01$ compared with non-COPD smokers. Statistical differences were determined with 1-way ANOVA followed by Bonferroni post-test.

$(23,24)$. To assess the role of Fbln1 in COPD pathogenesis, we generated mice deficient in the Fbln1c isoform using a gene-targeting strategy. A Fbln1c-specific targeting vector was generated (Figure 2, A-C) and transfected into embryonic stem cells (ES cells) to specifically delete this isoform. After injection of recombinant ES cells into C57BL/6 mice, the Fbln1c gene was deleted in the offspring, confirmed by Southern blot and qPCR analyses (Figure 2, D-G). $\mathrm{Fbln}_{1 \mathrm{C}^{-1-}}$ mice displayed no developmental abnormalities (unpublished observation) or baseline differences in airway remodeling or inflammation (Figure 3).

Deletion of Fbln1c in mice inhibits airway and lung remodeling and protects against experimental COPD. WT and Fbln1 $\mathrm{C}^{-1-}$ C57BL/6 mice were exposed to CS or normal air for 8 weeks, and the amount of collagen in lung tissue assessed. The decreases in total and soluble collagen levels, as well as Colla1 protein in experimental COPD, were prevented in $\mathrm{Fbln}_{1 \mathrm{C}^{-1}}$ mice (Figure 3, A and B). $\mathrm{Fbln}_{1} \mathrm{C}^{-1}$ mice were also completely protected from increased collagen deposition around the small airways (Figure 3, C and D and Supplemental Figure $2 \mathrm{~A}$ ). WT mice also had increased $\alpha$-smooth muscle actin-positive ( $\alpha$-SMA-positive) cells around the small airways after CS exposure, whereas $\mathrm{Fbln}_{1} \mathrm{C}^{-1-}$ mice did not (Figure 3E). Emphysema-like alveolar enlargement was partially inhibited in CS-exposed $F b l n 1 C^{-1}$ mice compared with WT mice (Figure 3F and Supplemental Figure 2B). Furthermore, CS-exposed Fbln $1 \mathrm{C}^{-1-}$ mice were also protected against changes in lung function, with no increase in lung volume (Figure 3G) or static lung compliance (Figure 3H) compared with WT controls.

Since global deletion of Fbln Ic suppresses COPD pathogenesis, we assessed whether intranasal treatment with a specific siRNA targeting $F b l n l c$ had therapeutic effects. Mice were administered $F b l n l c$ or scrambled siRNA every 2 days during acute (4-day) CS exposure or from weeks 6-8 of chronic (8-week) CS exposure. These are the periods when Fbln $1 c$ mRNA expression first increases and fibrosis emerges, respectively. The efficiency of Fbln1c knockdown by siRNA was tested in mouse lungs. siRNA treatment significantly reduced Fbln1c expression after acute and chronic CS exposure, but Fbln1d was unaffected (Supplemental Figure 3, A and B). Previous in vivo studies showed that siRNA localizes predominantly in peribronchial epithelial cells after intranasal administration to mice (25). Thus, Fbln1c was likely downregulated, at least in these cells. siRNA treatment completely inhibited chronic CS-induced decreases in total and soluble lung collagen and Col1a1 levels, which were restored to the levels in controls (Figure 4, A and B). There was a corresponding reversal of collagen deposition around small airways (Figure 4C). siRNA treatment also suppressed emphysema-like alveolar enlargement (Figure 4D and Supplemental Figure 3C). These improvements in pathological features also protected against changes in lung function (Figure 4, E and $\mathrm{F}$ ).

Fbln1c is required for interactions with its binding proteins to generate collagen. Fbln1 is important for ECM stabilization; therefore, its role in the deposition of collagen and tissue remodeling in experimental COPD was determined. Eight weeks of CS exposure of WT mice resulted in increases in protein levels of Fn, whereas Tnc remained the same in whole lung tissue (Figure 5A). These levels were significantly reduced in Fbln $1 c^{-1-}$ mice. Decreased CS-induced Postn protein levels in WT mouse lungs were restored to control levels in $F b \ln 1 c^{-1-}$ mice. Similarly, treatment with siRNA against $F b \ln 1$ during CS exposure also returned Fn and Postn to control levels but had no effect on Tnc protein amounts (Supplemental Figure 4).

We then determined the effects of Fbln1c on the distribution of the other ECM proteins during 8

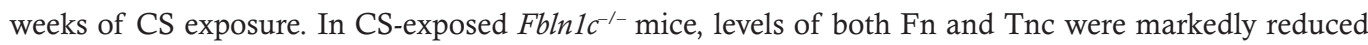
around the small airways, whereas Postn was increased compared with levels in CS-exposed WT controls (Figure 5, B-D and Supplemental Figure 5). Versican (Vcan), hyaluronan and proteoglycan link protein (Hapln1), and ECM1 protein levels were not altered in CS-exposed mice, nor were they dependent on the presence of Fbln1c (Supplemental Figure 6). 
A

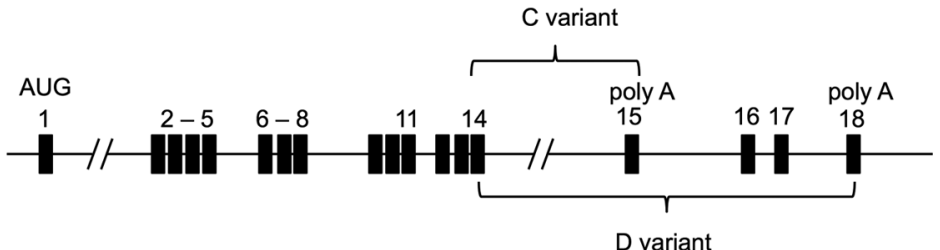

B

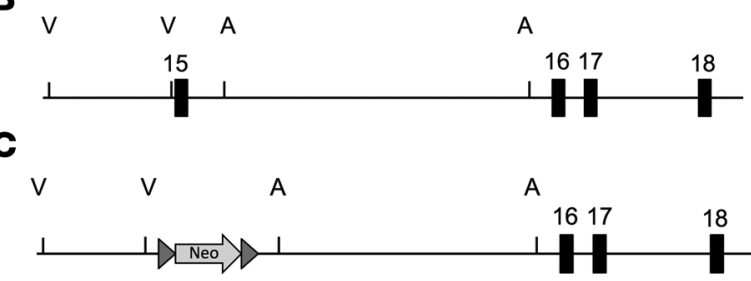

D

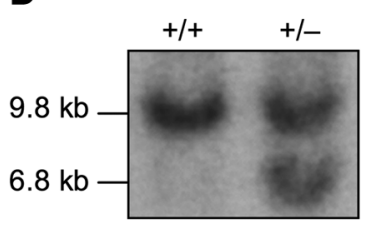

EcoRV
E

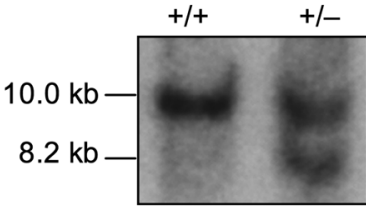

Apa I

\section{$\mathbf{F}$}

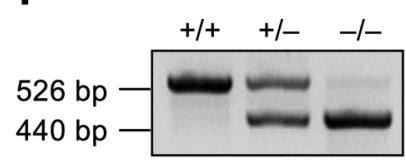

Figure 2. Generation of $\mathrm{Fb}_{\mathrm{n} 1 \mathbf{c}^{-/-}}$mice. (A) Mouse Fbln1 gene diagram with alternatively spliced exons that encode the C-terminal variable domains of the Fbln1c and $-d$ variants. (B) Expanded region of Fbln1 gene showing exons 15-18. (C) Targeted Fbln1 allele following recombination including the Neo cassette. Gray triangles indicate loxP sites used to remove the Neo cassette. Southern blots of genomic DNA from an ES cell clone transfected with exon 15 targeting vector. (D) Probe1 was hybridized to EcoRV digested genomic DNA. The 9.8-kb fragment is from the WT allele, and the 6.8-kb fragment is from the targeted allele. (E) Probe2 was hybridized to Apal digested DNA. The 10-kb fragment is derived from the WT allele and the $8.2 \mathrm{~kb}$ from the targeted allele. (F) PCR of genomic DNA from mice $^{+/+},+/-$, and ${ }^{-/-}$for the deletion of exon 15 and lacking the Neo cassette. (C) Reversetranscriptase PCR of RNA from hearts of E13.5 WT embryos and embryos heterozygous and homozygous for the targeted deletion.

G

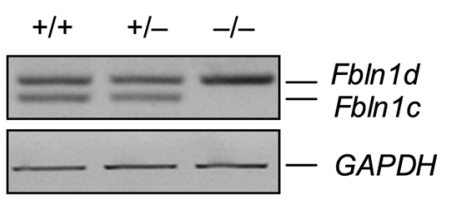

Next, we investigated the relationship between Fbln1, its interacting partners, and collagen around small airways after 8 weeks of CS exposure. Fbln1, Fn, Tnc, Postn, and Colla1 proteins colocalized around small airways after CS exposure and were markedly decreased in Fbln $1 \mathrm{C}^{-1-}$ mice (high magnification, Figure 5, E-G, low magnification Supplemental Figure 7-9).

Fbln1c promotes inflammation in experimental COPD. As ECM protein production and remodeling can affect inflammation (26), leukocyte responses and proinflammatory molecular signals were examined in CS-exposed WT and Fbln $1 \mathrm{c}^{-1-}$ mice.

Eight weeks of CS exposure resulted in increases in total leukocyte numbers dominated by macrophages and neutrophils in BALF in WT mice compared with normal air-exposed controls, whereas Fbln $1 \mathrm{c}^{-1-}$ mice showed significantly reduced numbers of these inflammatory cells (Figure 6A).

Eight weeks of CS exposure induced increases in the levels of proinflammatory cytokines TNF- $\alpha$ and IL-33, the fibrotic cytokine TGF- $\beta$, and the chemokine CXCL1 in the lung tissue of WT mice. Each of these factors is important in COPD pathogenesis (27). CS-exposed Fbln $1 \mathrm{C}^{-1-}$ mice had significant reductions in all of these factors in lung tissues (Figure 6, B-E). However, there were no differences in protein levels of these inflammatory factors in BALF between WT and $\mathrm{Fbln}_{1} \mathrm{c}^{-/-}$mice, whether they were exposed to CS or not (Supplemental Figure 10, A-C). In addition, TGF- $\beta$ was undetectable in BALF.

As genetic depletion of Fbln1c throughout CS exposure reduced inflammation, the therapeutic effect of siRNA knockdown in WT mice was assessed. Fbln1c siRNA knockdown led to a selective decrease in BALF neutrophils (Figure 6F). Fbln1c knockdown did not affect lung TNF- $\alpha$, IL-33, or TGF- $\beta$ protein levels; however, concomitant with the reduction in neutrophils, CXCL1 levels were selectively decreased (Figure 6, G-J). Therapeutic siRNA treatment did not affect BALF cytokine levels (Supplemental Figure 10, D-F).

Since the Smad family of proteins - particularly Smad2, -3 , and -4 - are important in downstream TGF- $\beta$ signalling (28), we measured the mRNA levels of these factors in the lungs of WT and Fbln $1 c^{-1-}$ mice after 8 weeks of CS exposure (Supplemental Figure 11). CS exposure did not affect Smad2 mRNA

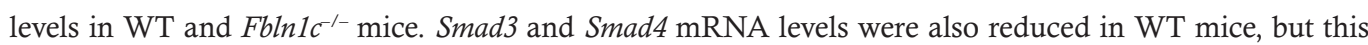
reduction did not occur in $\mathrm{Fb} \ln 1 \mathrm{C}^{-1-}$ mice. 
A

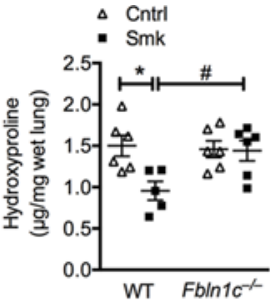

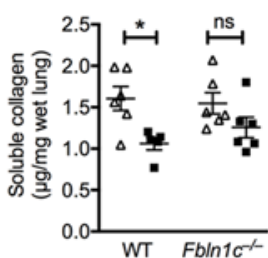

B

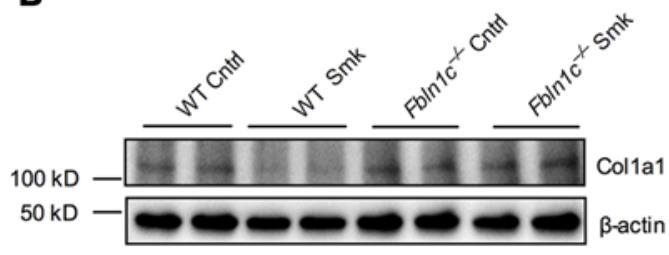

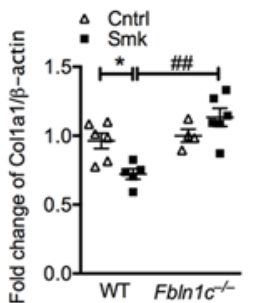

E
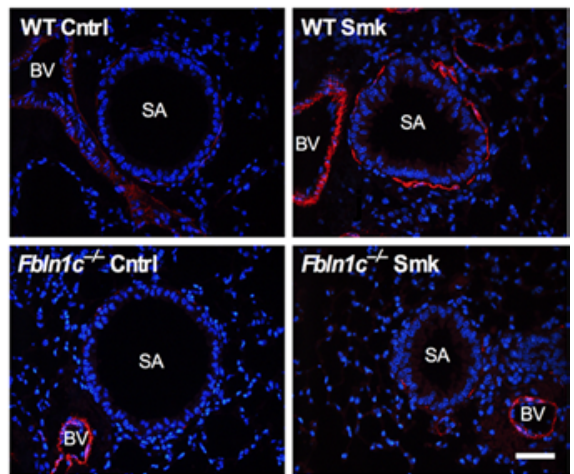

H

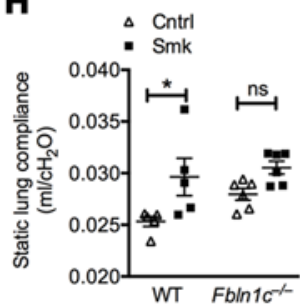

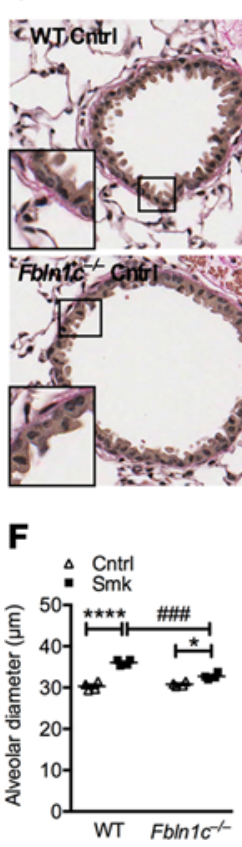

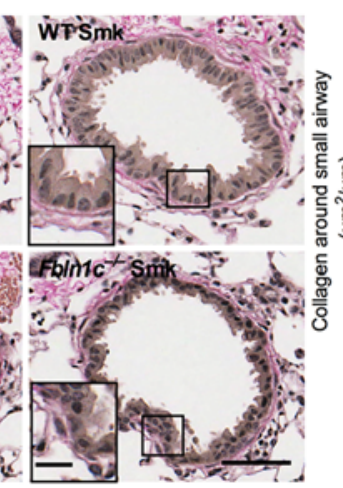

G

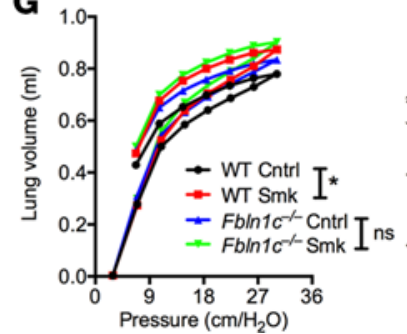

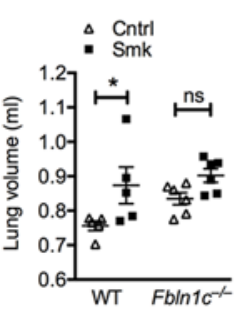

D

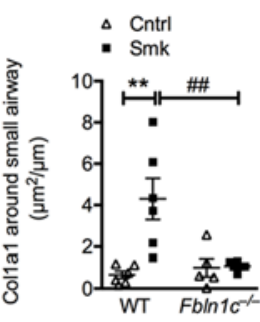

Figure 3. Absence of Fbln1c protects against airway and lung remodeling, emphysema-like alveolar enlargement, and impaired lung function in experimental COPD. WT and Fbln1 $\mathrm{c}^{-1-}$ mice were exposed to cigarette smoke (CS) for 8 weeks to induce experimental COPD; controls were exposed to normal air. (A) Total (left) and soluble collagen (right) in whole lungs. $n=5-6$. (B) Type I collagen (Col1a1) protein in whole lung tissues assessed by immunoblot (left), and fold change of densitometry normalized to $\beta$-actin (right). $n=5-6$. (C) Collagen deposition around small airways in mouse lung sections stained with Verhoeff's-Van Gieson (VVG, left; scale bar: $50 \mu \mathrm{m}$; inserts show expanded image of indicated regions; scale bar: $15 \mu \mathrm{m}$ ), and quantification is normalized to the perimeter of basement membrane (Pbm, right). $n=24-40$ airways from $n=4-6$ mice per group. (D) Col1a1 area around mouse small airways normalized to the Pbm. $n=24-40$ airways from $n=4-6$ mice per group. (E) $\alpha$-Smooth muscle actin-positive (SMA-positive) cell (red) and nuclear staining (hoechst, blue) around small airways by immuofluorescence (top; SA, small airway; BV, blood vessel; scale bar: $50 \mu \mathrm{m}$ ), and quantification is normalized to the Pbm (bottom). $n=24-40$ airways from $n=4-6$ mice per group. (F) Emphysema-like alveolar enlargement was measured by assessment of alveolar diameter. $n=5-6$. Lung function was measured in terms of (G) pressure-volume loops and peak volumes and (H) static lung compliance. $n=5-6$. Results are mean \pm SEM. ${ }^{*} P<0.05$, ${ }^{* *} P<0.01$, ${ }^{* * * *} P<0.0001$ compared with normal air-exposed WT or Fbln1c ${ }^{-/-}$

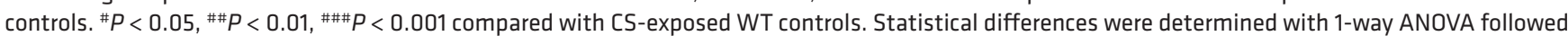
by Bonferroni post-test.

Deletion of Fbln1c in mice inhibits airway and lung remodeling in chronic asthma and lung fibrosis. Since airway inflammation and remodeling are also important features of other chronic lung diseases, including asthma and IPF, the effect of the absence of Fbln1c on experimental models of these diseases was assessed. Mice were chronically treated with HDM extract intranasally for 5 days per week for 5 weeks, which resulted in increased lung inflammation (low magnification, Figure 7A) and collagen deposition around airways in WT mice (high magnification). In other groups, bleomycin-induced lung fibrosis was induced and assessed 28 days later (lung fibrosis at low magnification and collagen deposition around airways at high magnification, Figure 7B). In both models, collagen deposition around small airways was completely inhibited in $\mathrm{Fbln} 1 \mathrm{c}^{-1-}$ mice.

\section{Discussion}

The respiratory diseases COPD, severe asthma, and IPF are among the most common and serious human diseases of today. They are difficult to treat, and there are no currently available broadly effective treatments. The identification of novel therapeutic targets may lead to the development of new treatments. Increased 
A $\triangle \mathrm{Cntrl}+\mathrm{dH}_{2} \mathrm{O}$

- Smk $+\mathrm{dH}_{2} \mathrm{O}$

- Smk + scram siRNA

- Smk + Fbln1c siRNA

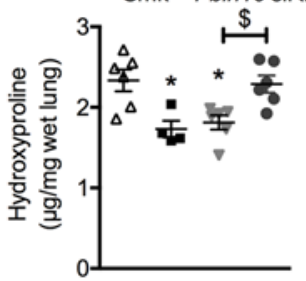

B

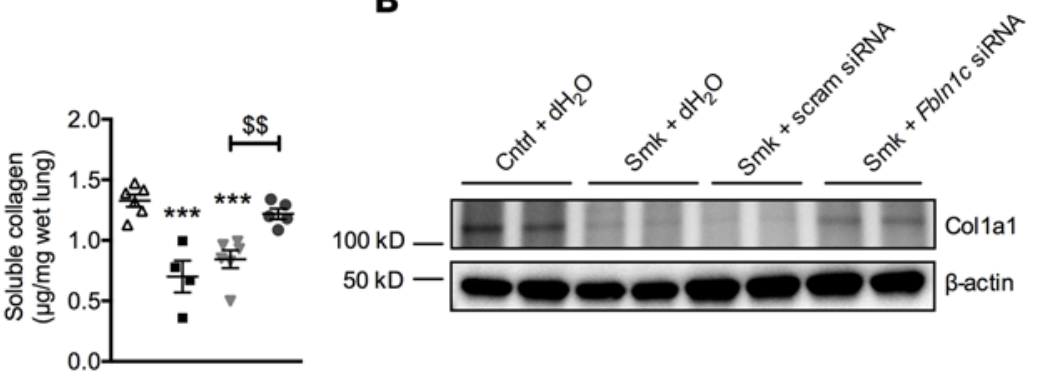

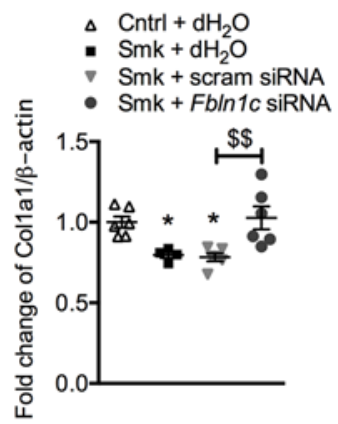

- Smk + Fbln1c siRNA

C

Cntrl $+\mathrm{dH}_{2} \mathrm{O}$

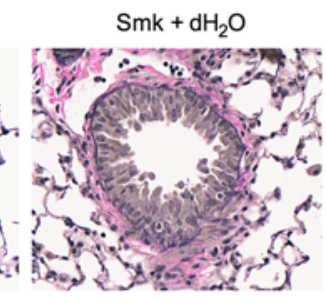

Smk + scram siRNA
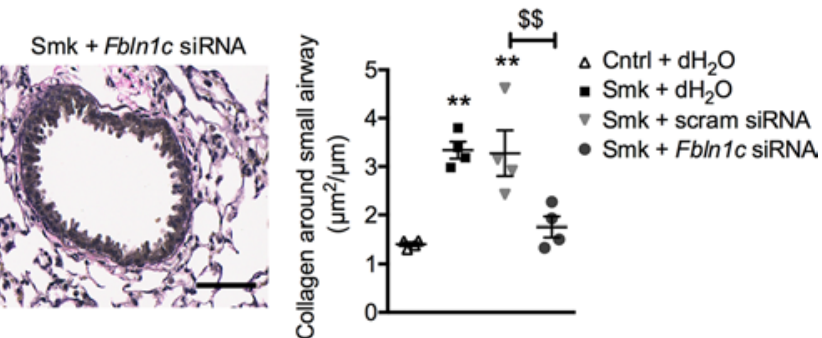

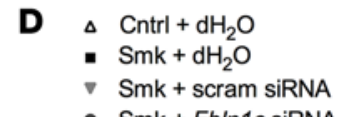

v $\mathrm{Smk}$ + scram siRNA

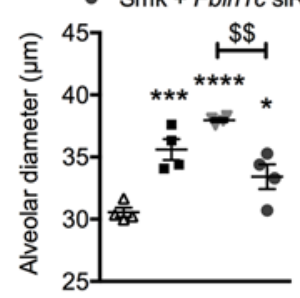

E

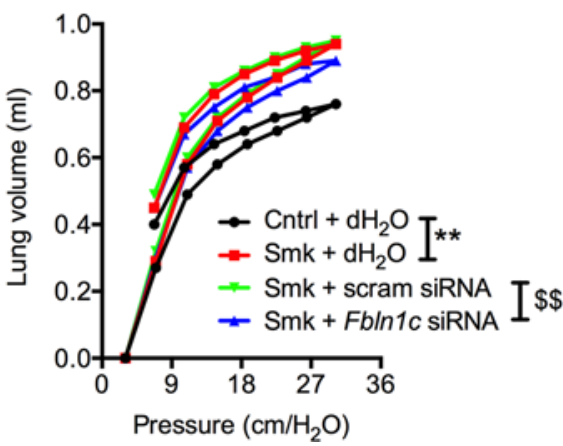

$\Delta$ Cntrl $+\mathrm{dH}_{2} \mathrm{O}$

- $\mathrm{Smk}+\mathrm{dH}_{2} \mathrm{O}$

- Smk + scram siRNA

- Smk + Fbln1c siRNA

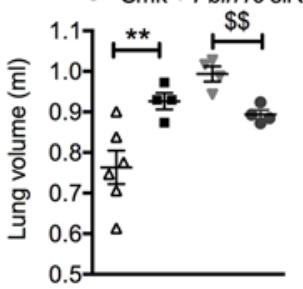

$\mathbf{F}$
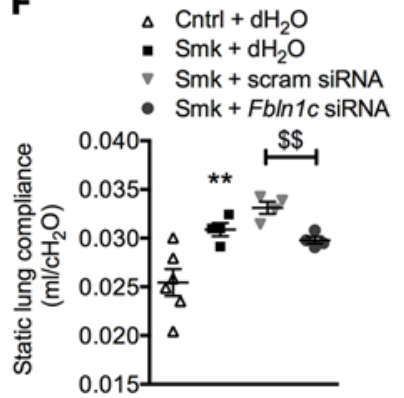

Figure 4. FbIn1c siRNA protects against airway and lung remodeling, emphysema-like alveolar enlargement, and impaired lung function in experimental COPD. WT mice were exposed to CS for 8 weeks to induce experimental COPD and were treated with Fbln1c or scrambled siRNA. (A) Total (left) and soluble collagen (right) in whole lungs. $n=4-6$. (B) Col1a1 protein in whole lungs (left), and fold change of densitometry normalized to $\beta$-actin (right). $n=4-6$. (C) Collagen stained with VVG (left; scale bar: $50 \mu \mathrm{m}$ ) and normalized to Pbm (right). $n=4-6$. (D) Emphysema-like alveolar enlargement. $n=4-6$. Lung function in terms of (E) pressure-volume loops and peak volumes and (F) static lung compliance. $n=4-6$. Results are mean $\pm S E M$. ${ }^{*} P<0.05$, ${ }^{* *} P<0.01,{ }^{* *} P<0.001,{ }^{* * *} P<0.0001$ compared with normal air-exposed WT; ${ }^{\$} P<0.05$, $\$ \$ P<0.01$ compared with CS-exposed controls treated with scrambled siRNA. Statistical differences were determined with 1-way ANOVA followed by Bonferroni post-test.

production of ECM proteins can have serious pathological consequences in these and other diseases. Here, we discover important roles for the ECM protein Fbln1 in airway and lung remodeling, and also in driving inflammation in COPD. Fbln1 protein is elevated in pBECs and serum from COPD patients and in a chronic CS-induced mouse model of experimental COPD. Genetic or therapeutic inhibition of Fbln1c reversed decreases in collagen in lung parenchyma, likely as a result of protecting against emphysema in CS-exposed WT mice. It also inhibited the increase in collagen deposition around the small airways in experimental COPD. Fbln1c also contributes to chronic inflammation, as $F b \ln 1 \mathrm{C}^{-1-}$ mice were protected against CS-induced inflammatory cell influx into BALF and proinflammatory cytokine and chemokine production in the lungs. Accordingly, lung function was improved in CS-exposed Fbln $1 \mathrm{C}^{-1-}$ mice. Thus, targeting Fbln1c suppressed hallmark features of airway and lung remodeling, emphysema, and inflammation and improved lung function in experimental COPD. It also suppressed airway remodeling in experimental chronic asthma and pulmonary fibrosis.

We found increased levels of Fbln1 in pBECs and serum from COPD patients, as well as in the airway epithelium and lung tissue in experimental COPD. This extends other observations in which Fbln1 was increased in the serum of asthma (13) and IPF patients (15), suggesting that Fbln1 may be a biomarker and therapeutic target in respiratory diseases and other conditions involving remodeling and inflammation. 
A
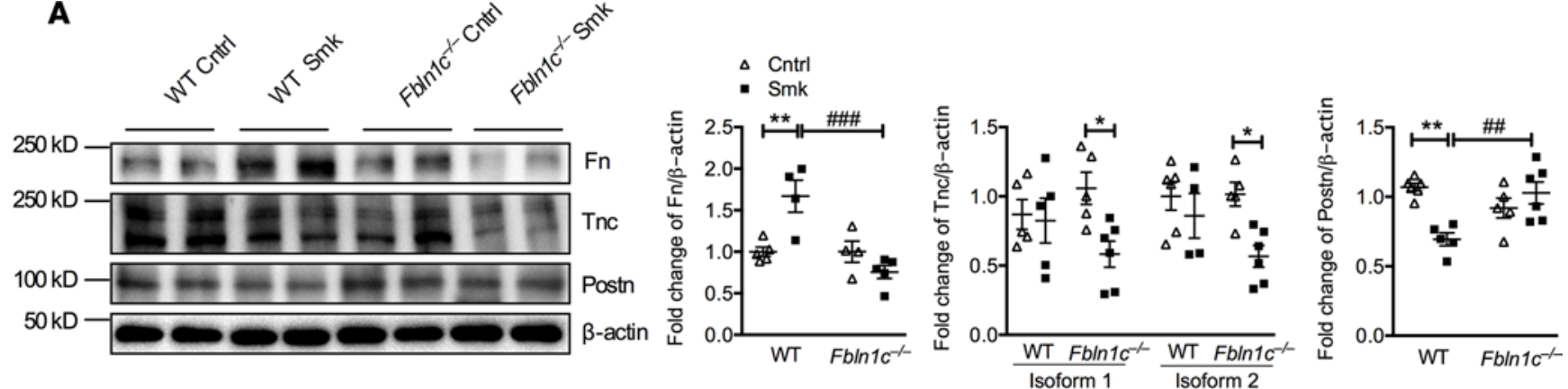

B

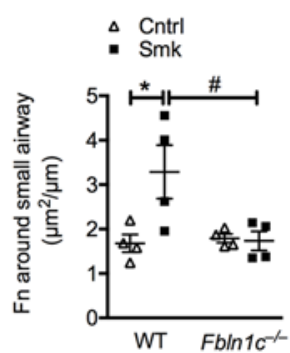

E
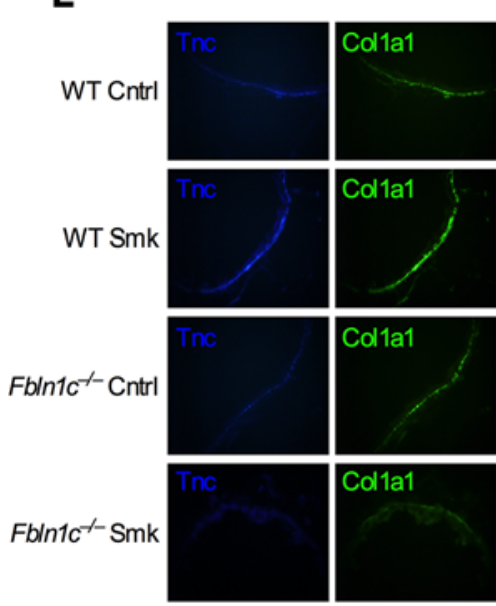

G
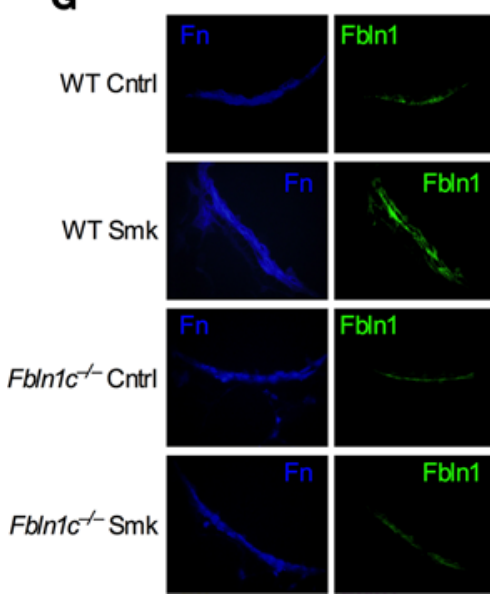

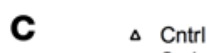

- Smk

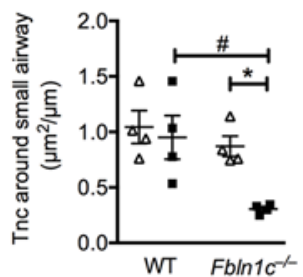

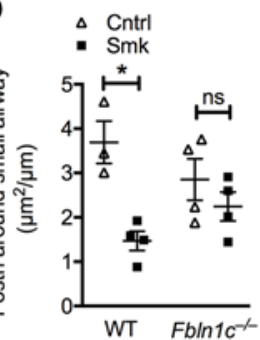

$\mathbf{F}$
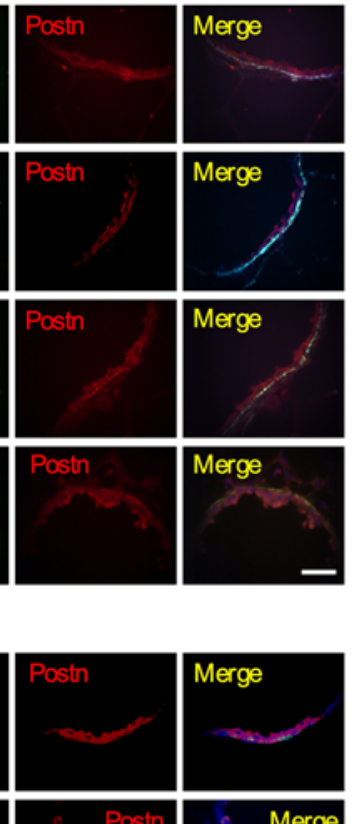

Fbln1c ${ }^{-1-} \mathrm{Cntr}$
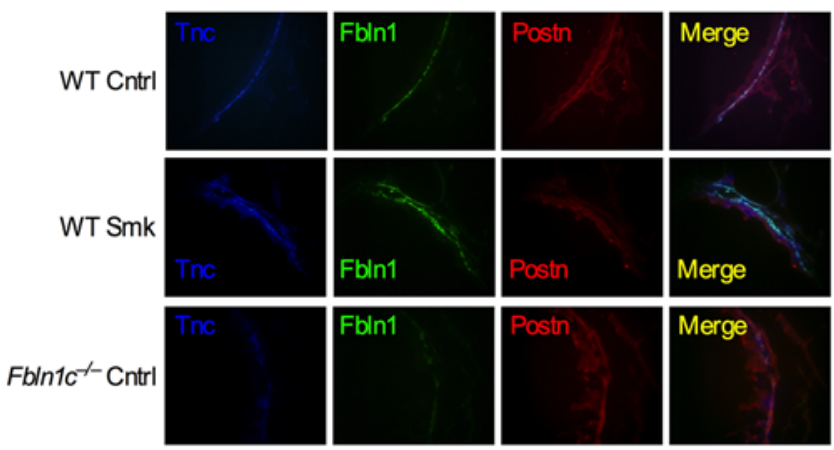

Fbln1c ${ }^{-1-}$ Smk
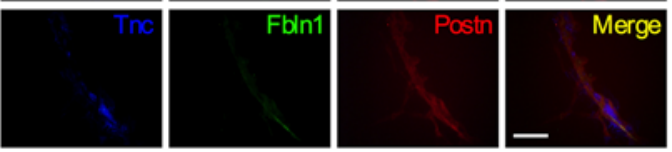

Figure 5. Fbln1c binding proteins are altered in whole lungs and around small airways in WT and $\mathrm{Fbln1c}^{-/-}$mice with experimental COPD. WT and Fbln1 $\mathrm{C}^{-/-}$mice were exposed to cigarette smoke for 8 weeks to induce experimental COPD; controls were exposed to normal air. (A) Fibronectin (Fn), tenascin-c ( $T n c)$, and periostin (Postn) protein levels in whole lungs assessed by immunoblot (left), and fold change of densitometry normalized to $\beta$-actin (right). (B) Fn, (C) Tnc, and (D) Postn area around the small airways analyzed by IHC and normalized to perimeter of basement membrane (Pbm). $n=24-40$ airways from $n=$ 5-6 mice per group. (E) Colocalization of Tnc (blue), Col1a1 (green), and Postn (red) around the small airways. (F) Colocalization of Tnc (blue), Fbln1 (green), and Postn (red). (G) Colocalization of Fn (blue), Fbln1 (green), and Postn (red) (scale bar: $15 \mu \mathrm{m} . n=3$.). Results are mean \pm SEM. ${ }^{*} P<0.05,{ }^{* *} P<0.01$ compared with air-exposed WT or Fbln1c ${ }^{-1-}$

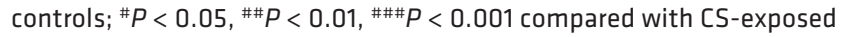
WT controls. Statistical differences were determined with 1-way ANOVA followed by Bonferroni post-test.

We did not observe altered Fbln1 protein levels in primary fibroblasts from excised lungs from mild to moderate COPD patients compared with non-COPD controls with lung cancer (data not shown). A more in-depth study of fibroblasts that includes the examination of non-COPD, non-lung cancer controls is needed to clarify the roles of these cells in Fbln-related events. Several types of mesenchymal cells are 
A

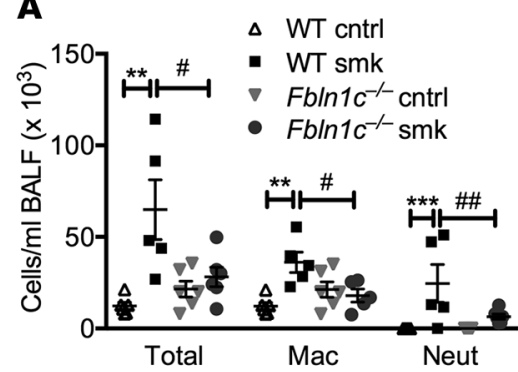

$\mathbf{F}$

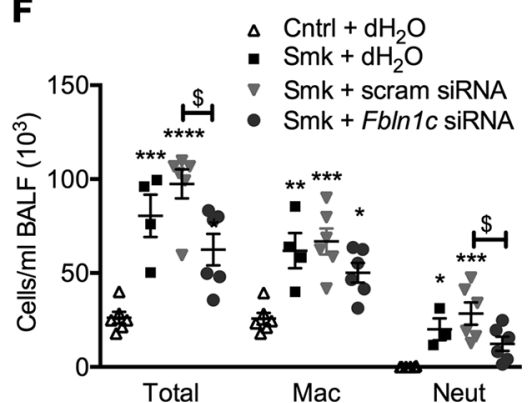

B

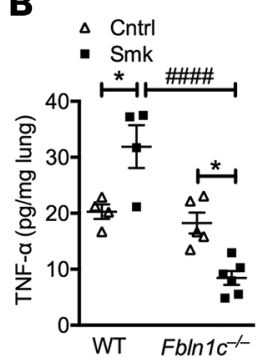

G $\begin{aligned} \Delta & \mathrm{Cntrl}+\mathrm{dH}_{2} \mathrm{O} \\ & \text { - } \mathrm{Smk}+\mathrm{dH}_{2} \mathrm{O}\end{aligned}$

- Smk + scram siRNA

- Smk + Fbln1c siRNA

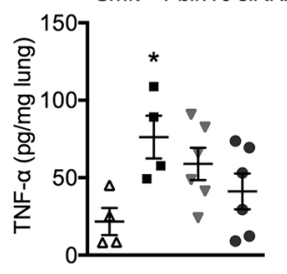

C

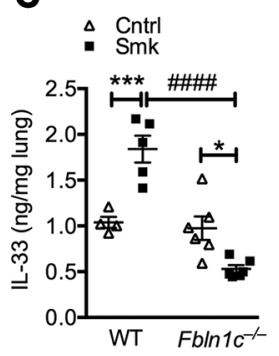

$\begin{aligned} \text { H. } & \mathrm{Cntrl}+\mathrm{dH}_{2} \mathrm{O} \\ \text { - } & \mathrm{Smk}+\mathrm{dH}_{2} \mathrm{O} \\ & \mathrm{Smk}+\text { scram siRNA } \\ & -\mathrm{Smk}+\text { Fbln1c siRNA }\end{aligned}$

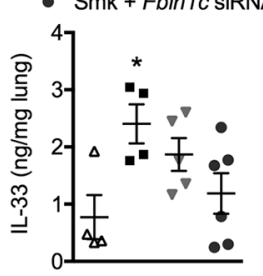

D

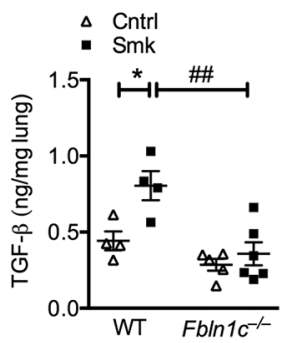

E

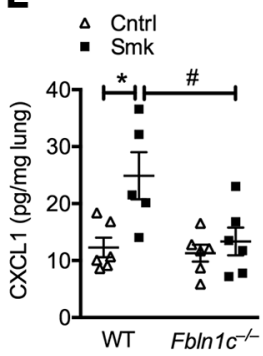

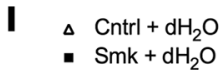

- Smk + scram siRNA

- Smk + Fbln1c siRNA

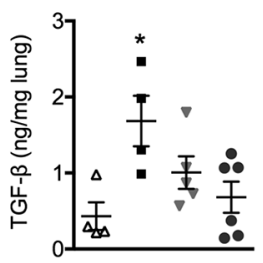

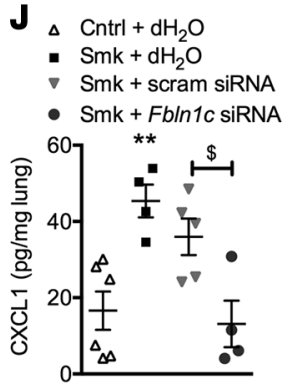

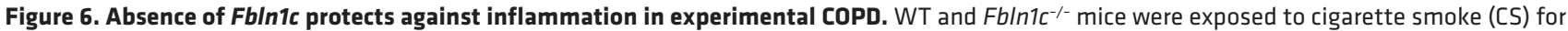
8 weeks to induce experimental COPD; controls were exposed to normal air. (A) Differential inflammatory cell counts in bronchoalveolar lavage fluid (BALF). (B) TNF- $\alpha,(\mathbf{C})$ IL-33, (D) TCF- $\beta$, and (E) CXCL1 protein in whole lungs measured by ELISA. WT mice were treated with Fbln1c or scrambled siRNA from weeks 6-8 of 8 weeks of CS exposure. (F) Differential inflammatory cell counts in BALF. (G) TNF- $\alpha$, (H) IL-33, (I) TGF- $\beta$, and (J) CXCL1 protein in whole lungs. Results are mean \pm SEM. ${ }^{*} P<0.05,{ }^{* *} P<0.01,{ }^{* *} P<0.001,{ }^{* * *} P<0.0001$ compared with normal air-exposed WT or Fbln1c ${ }^{-/-}$controls; ${ }^{\#} P<0.05,{ }^{\# \#} P<0.01$, \#\#\# $P<0.0001$ compared with CS-exposed WT controls; ${ }^{\$} P<0.05$ compared with CS-exposed controls treated with scrambled siRNA. Statistical differences were determined with 1-way ANOVA followed by Bonferroni post-test.

capable of producing Fbln1, and in the airways and lungs, there is likely not a single cellular source. In previous studies, we showed that ASM cells (13) and fibroblasts $(14,17)$ produce Fbln1. We also showed that Fbln1 is not upregulated by TGF- $\beta$ (17), but TGF- $\beta$-stimulated fibroblasts can incorporate exogenously produced Fbln1 into the ECM. Furthermore, Fbln1c1 peptide promotes the production of new Fbln1c1 in fibroblasts (14). Taken together, our studies indicate that soluble Fbln1c may be produced by cells, such as epithelial cells, and is incorporated into the airway by fibroblasts, especially when levels of profibrotic stimuli are increased, as occurs in COPD. This newly incorporated Fbln1 can then act as a stimulus for further ECM deposition, establishing a cycle of persistent fibrosis. This could be interrupted with therapeutic intervention.

Eight weeks of CS exposure to induce experimental COPD increased the levels of protein but decreased mRNA expression of Fbln1 in lung tissues. These discrepancies could be due to numerous factors, including alterations in DNA methylation, mRNA stability, and microRNA regulation. Our previous studies show that TGF- $\beta$ downregulated Fbln1 mRNA in ASM cells and that Fbln1 protein was translationally controlled (17). We also found that TGF- $\beta$ induced sequestration of soluble Fbln 1 into the ECM, rather than regulating de novo synthesis of Fbln1. Smads are important downstream factors in TGF- $\beta$ signaling pathways. Smad3 and Smad4 are downregulated in primary fibroblasts from COPD patients after CS extract challenge, whereas they are not changed in these cells from healthy controls (28). Other studies show that Smad3 is decreased in airway epithelial and stromal cells from COPD patients compared with controls (29) and Smad3-null mice develop spontaneous emphysema (30). We demonstrate in this study that WT mice with experimental COPD had reduced Smad3 and Smad4, whereas Fbln1 $c^{-1-}$ mice did not. This may explain why emphysema-like alveolar enlargement did not occur in $\mathrm{Fbln}_{1} \mathrm{c}^{-1-}$ mice exposed to CS for 8 weeks, although the exact mechanisms involved remain unclear.

Increases in Fbln 1 protein were associated with pulmonary remodeling, emphysema, and inflammation, which were reduced in Fbln $1 c^{-1-}$ mice. This shows that CS affects Fbln 1 homeostasis, increasing its production and likely modifying its degradation and peptide generation. Fbln1c peptides can stimulate Fbln1 deposition in COPD fibroblasts - potentially through a feed-forward mechanism — and promote 
A
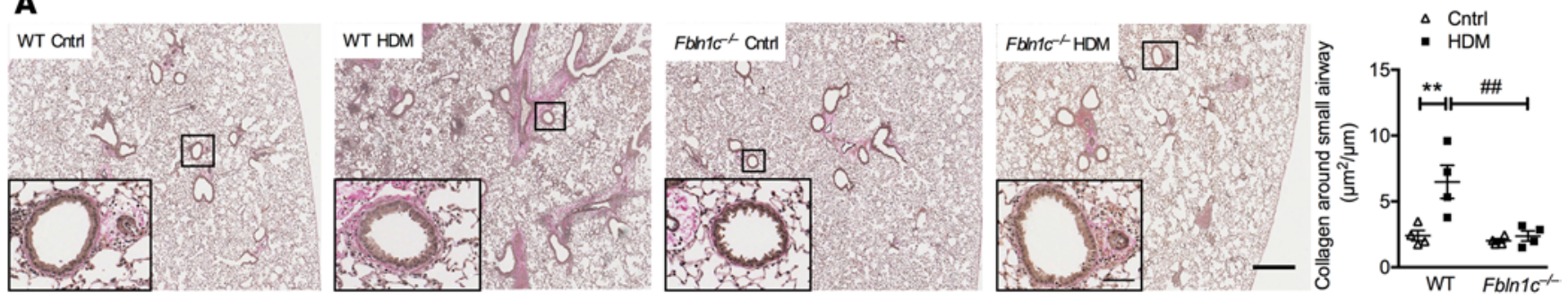

B
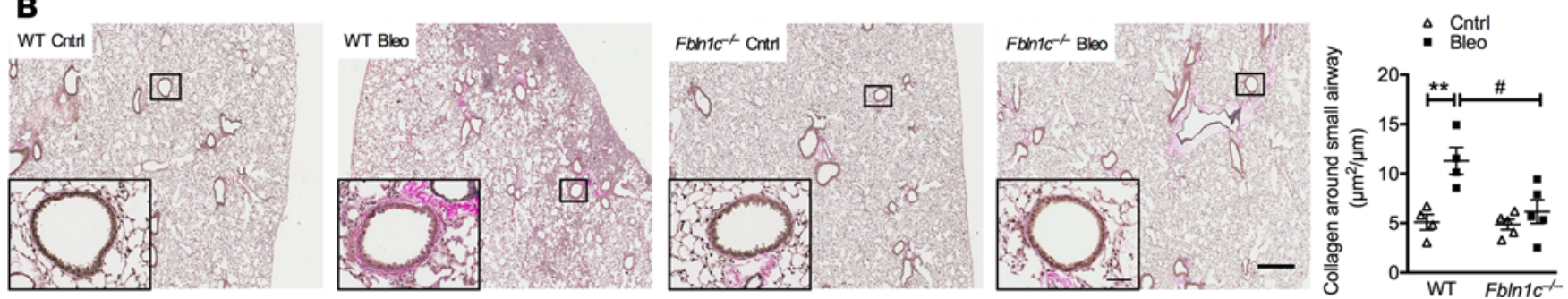

Figure 7. Absence of Fbln1c protects against airway remodeling in experimental chronic asthma and pulmonary fibrosis. WT and $F b / n 1 c^{-1-}$ mice were administered house dust mite (HDM) extract for 5 days per week for 5 weeks; controls were administered sterile saline. (A) Collagen deposition around small airways in lung sections stained with Verhoeff's-Van Gieson stain (VVG, left; scale bar: $500 \mu \mathrm{m}$ ). Inserts show expanded image of indicated regions (scale bar: $50 \mu \mathrm{m}$ ). Quantification of collagen deposition normalized to the perimeter of basement membrane (Pbm) (right). $n=24-40$ airways from $n=6-8$ mice per group. WT and $\mathrm{Fbln1}^{-1-}$ mice were administered bleomycin sulphate once, controls were administered sterile PBS, and tissues were collected 28 days later. (B) Collagen deposition around small airways and normalized to Pbm (scale bar: $500 \mu \mathrm{m}$ ). Inserts show expanded image of indicated regions (scale bar: $50 \mu \mathrm{m}) . n=24-32$ airways from $n=8$ mice per group. Results are mean \pm SEM. ${ }^{* *} P<0.01$ compared with WT or Fbln1 $c^{-1-}$ controls; ${ }^{\#} P<0.05$, \#\# $P<0.01$ compared with HDM or bleomycin-treated WT controls. Statistical differences were determined with 1-way ANOVA followed by Bonferroni post-test.

the attachment of ASM cells and fibroblasts; they also augment Fn and perlecan deposition (14). Some proteases, such as MMP13, cleave Fbln1 $(31,32)$, and the mRNA expression of this enzyme is upregulated in CS-exposed mice (Supplemental Table 1). Thus, there is the potential that increases in MMP13 and Fbln1 levels promote airway remodeling but also produce more cleavage products, inducing inflammation, as is the case with other ECM factors (33). It is possible that the increase in MMP13 was insufficient to control the levels of Fbln1c produced in response to CS exposure. However, this would result in the release of greater levels of fragments of Fbln1c that may further increase inflammatory responses. There were no differences in mRNA expression of MMP13 in the lungs between CS-exposed WT and Fbln1c ${ }^{-1-}$ mice, and the role of this protease needs further study. The calcium-binding protein calumenin protects Fbln1 from MMP13 cleavage, which in turn suppresses the phosphorylation of extracellular signal-regulated kinases 1 and 2 (ERK1/2) and cell migration (32). However, calumenin expression was not altered in CS-exposed mice (data not shown), suggesting that it is unlikely to be protecting against Fbln1 degradation. Thus, although the exact mechanisms are unknown, it is likely that Fbln 1 is constantly being produced and degraded, and when in excess, this leads to remodeling and inflammation.

To further assess mechanisms, primary fibroblasts from WT and Fbln $1 \mathrm{C}^{-1-}$ mice were isolated and exposed to CS extract. Supernatants were collected and added to cultures of BM-derived macrophages from WT and Fbln $1 \mathrm{C}^{-1-}$ mice. The release of cytokines and chemokines (TNF- $\alpha$ and CXCL1) was assessed, but we found no differences in response to exposure or between mouse strains. Microarray and proteomics analyses could be employed to further investigate the mechanisms involved, along with the ways in which the Fbln1c signal is sensed and how a response program is initiated, which would be important future directions. Furthermore, a more detailed dissection of the synthesis, secretion, and turnover of the associated ECM elements in the airway and parenchyma would facilitate the elucidation of how Fbln1c stabilizes collagen.

Fibrillar collagen is a major structural component of ECM and is important in maintaining tissue integrity (34). We observed CS-induced reductions in collagen levels in the parenchyma, which was Fbln1 dependent and likely contributes to loss of alveolar tissue. Human studies using microcomputed tomography show that the amount of collagen in lung tissue and respiratory bronchioles - the regions associated with emphysema - decreases in patients with more severe COPD (35). This suggests that 


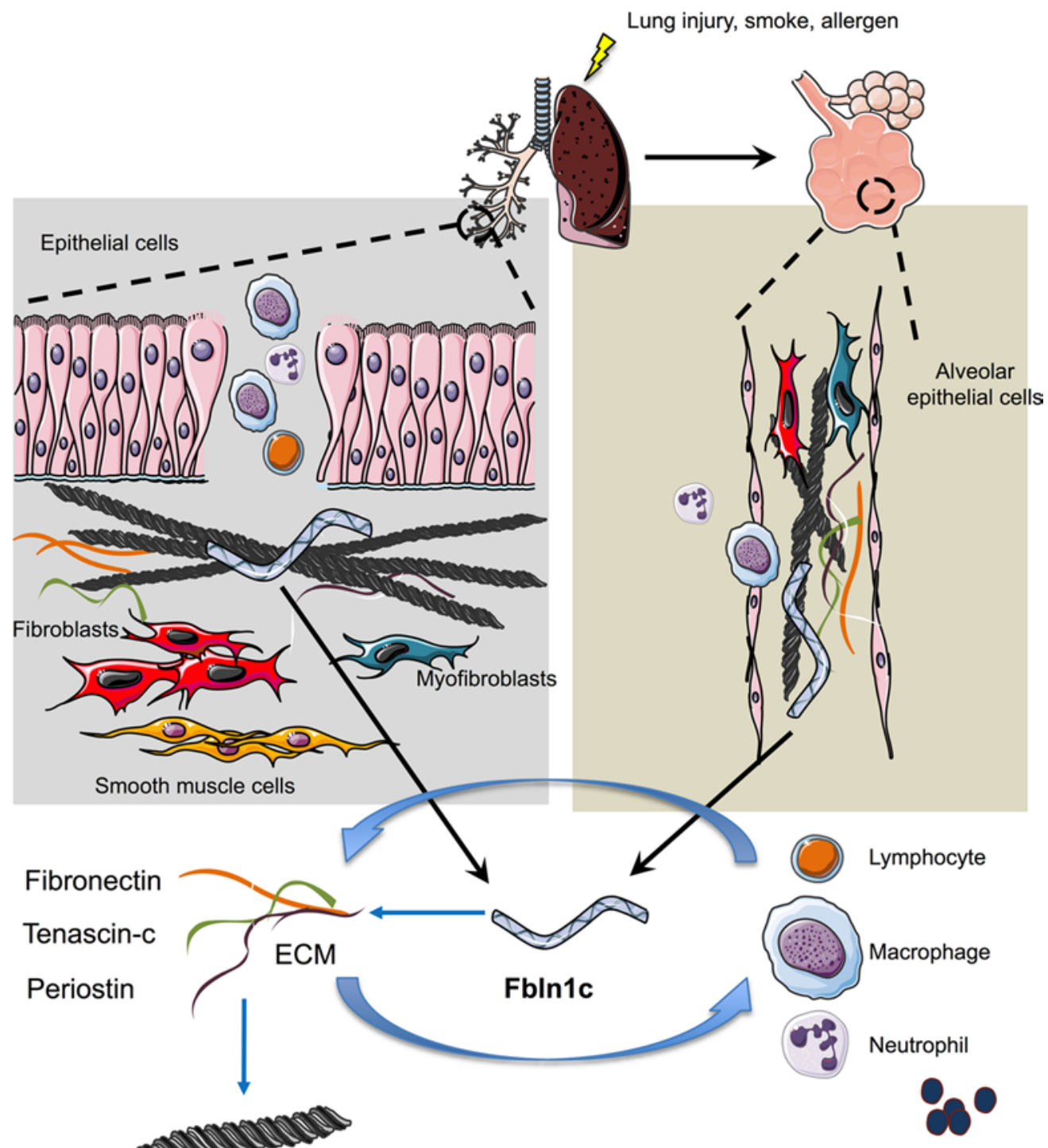

Collagen

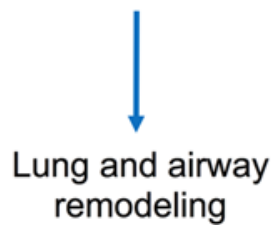

Cytokines/Chemokines

Figure 8. Schematic diagram of the role of Fbln1c in lung remodeling and inflammation. Lung injury, smoke, or allergen exposure increases Fbln1c in airways and parenchyma. This stabilizes fibronectin, tenascin-c, and periostin and promotes collagen deposition. Excess collagen deposition induces lung and airway remodeling and impaired lung function. Fbln1c also promotes airway inflammation - involving the influx of macrophages, neutrophils, and lymphocytes - and increases in associated cytokines/chemokines that induce the production of ECM proteins and the development of respiratory disease.

alveolar collagen loss increases with COPD severity. Since mice do not have respiratory bronchioles, the similar decreases in collagen in mice and humans likely occur in the terminal bronchioles.

Paradoxically, we found Fbln1-dependent increases in collagen around the small airways in experimental COPD. A similar phenomena occurs in the lungs in severe COPD, where regions of bronchiolar tissue destruction are closely associated with the thickening of airway tissue (35). It is surmised that fibrotic repair mechanisms are initially induced in both tissues; however, chronic stimulation results in a switch to an antifibrotic phenotype in the parenchyma only. Thus, inverse alterations in collagen levels in different 
tissues may reflect advanced progression of disease. Generally, acute CS exposure increases the expression of profibrotic mediators in both the parenchyma and airways (36). Chronic exposure induces continuous low-grade inflammation and reduces gene expression of matrix proteins and positive regulators of matrix formation in the parenchyma only (36). This suggests that lung tissue is uniquely prone to these geneexpression changes and emphysema development only after chronic CS exposure.

Thus, Fbln $1 \mathrm{C}^{-1-}$ mice are protected against abnormal deposition of collagen in lungs in experimental COPD, although the mechanisms involved are unclear. In other assays, we found that the expression of the common collagenases (MMP1, -3, and -8) and proteases (MMP7, -12, and -13) were not Fbln1c dependent (Supplemental Figure 12). Nevertheless, many other collagenases and proteases are present in the lungs, and their involvement requires further investigation. It is likely that unknown molecular and cellular factors that may have site-specific actions may also contribute to different effects in different tissues. This could be resolved by microdissection and omics analyses of different tissues in WT and Fbln $1 \mathrm{C}^{-1-}$ mice with and without CS exposure. However, this is beyond the scope of the current study.

To maintain lung structure and induce fibrosis, ECM proteins need to interact with each other in the correct confirmation. Our study shows that Fbln 1 critically regulates these interactions during excess fibrosis in 3 respiratory diseases (Figure 8). Fbln1 directly interacts with other ECM proteins, including Fn $(32,37)$. Fbln1 binds to the Heparin II domain (38) and has cell-adhesion and motility-suppressive effects on Fn-coated substrates (39). Tnc has a binding domain similar to that of Fbln1 and could bind with Fn (40). Furthermore, Postn and Fn bind to collagen, and Fbln1, Tnc, and Postn colocalize in skin keratinocytes $(11,41,42)$. Fn, Tnc, and Postn have altered protein expression in $\mathrm{Fbln}_{1} \mathrm{c}^{-1}$ mice, indicating that Fbln1 directly or indirectly binds to multiple targets and organizes collagen structure in COPD. This may result from the dysregulation of RNA or altered secretion/deposition. However, as the "matrisome" that makes up and regulates ECM is extensive (>1,000 proteins) (43), other proteins are likely involved, and their elucidation would be a major undertaking and requires further research.

Alterations in individual ECM proteins changes the overall physical properties of the matrix affecting cell movement and function, local macromolecule activity, and the ability to bind cytokines and contribute to inflammation. In chronically inflamed tissues, such as COPD-patient lungs, aberrant ECM protein expression and fragment generation affects cellular motility and promotes immune responses (26). Collagenderived peptide fragments, in particular proline-glycine-proline (PGP), are increased in the lungs of COPD patients and contribute to inflammation through the chemoattraction of neutrophils (44). The stabilization of collagen levels in $\mathrm{Fbln}_{1 \mathrm{C}^{-1}}$ mice may contribute to the reduction in neutrophil influx and numbers.

Previous studies demonstrated that remodeling and inflammation are interdependent (45). We consider that Fbln1 induces remodeling and inflammation likely through such interdependent processes that, in combination, promote the phenotype observed. We demonstrate here that $\mathrm{Fbln}_{1} \mathrm{C}^{-1-}$ mice have reduced cellular inflammation in the airways and decreased levels of the proinflammatory cytokines TNF- $\alpha$ and IL-33; a profibrotic cytokine involved in airway remodeling $(46,47)$; and the chemokine CXCL1 in the lungs. This may be the result of a direct effect on cellular expression of these cytokines and/or chemokines, or it may occur indirectly through reductions in other immunomodulatory factors that control their expression, such as Tnc. Alterations in other ECM proteins also affects inflammation. Tnc $^{-1-}$ and Postn $^{-1-}$ mice have reduced inflammation in models of asthma and lung fibrosis, respectively $(48,49)$. Tnc is regulated by both the spatial and temporal distribution of Fbln in chronic contact dermatitis (50) and is an activator of TLR4-mediated immunity that facilitates persistent inflammation and tissue destruction in arthritic joint disease (51). Thus, the reduced levels of Tnc shown here in both the parenchyma and airways in CS-exposed $\mathrm{FblnIC}^{-1-}$ mice could contribute to the decreases in TNF- $\alpha$ and CXCL1 production, affecting neutrophil chemotaxis.

Airway remodeling is an important feature of other respiratory diseases, including asthma and IPF. We show that $\mathrm{Fbln}_{1 \mathrm{C}^{-1}}$ mice have reduced collagen deposition around the airways in chronic allergic airways disease and lung fibrosis mouse models. Experiments determining the mechanisms involved in these models are currently ongoing. This shows that Fbln 1 is a critical mediator of fibrosis in multiple models of airway remodeling induced by different factors.

Our data show that the Fbln1c isoform affects remodeling and inflammation in respiratory diseases. Unlike mice, humans also have Fbln1a and -b. Fbln1 has 3 domains (I, II, and III). All Fbln1 variants contain domain I and II, and the only difference is their domain III, which is localized in their C-terminus (52). Fbln1a does not have the C-terminal domain and that domain in Fbln $1 \mathrm{~b}$ is shorter and therefore is 
likely less functional than in Fbln1c. Furthermore, Fbln1a and -b are developmentally expressed and are not typically found in adults. Thus, it is unlikely that Fbln1a and -b compensate for Fbln1c in humans. Fbln1d also does not appear to compensate, since its expression did not increase when Fbln1c was inhibited with siRNA in mice (Supplemental Figure 1C).

Collectively, our data show that Fbln1, especially Fbln1c, plays important roles in the pathogenesis of COPD. Fbln 1 regulates airway and parenchymal collagen deposition by organizing ECM proteins, promoting airway remodeling and emphysema; it also induces inflammation, and these events lead to a reduction in lung function. Fbln1 also promotes airway remodeling in other models of chronic respiratory disease. This identifies Fbln1 as a potential therapeutic target in chronic airway and potentially other fibrotic and inflammatory diseases and warrants further investigation.

\section{Methods}

Human subjects. Nine patients were recruited with stage III severe COPD with forced expiratory volume in 1 second $\left(\mathrm{FEV}_{1}\right) 30 \%-50 \% \mathrm{COPD}$ and classified according the Global Initiative for Chronic Obstructive Lung Disease (GOLD) criteria (Supplemental Table 2) (53). They were defined by fixed airflow limitation on spirometry with an $\mathrm{FEV}_{1}$ / forced vital capacity (FVC) ratio less than $70 \%$, and $\mathrm{FEV}_{1}$ less than $80 \%$ predicted. All were ex-smokers (at least 1 year abstinent) and none were using inhaled corticosteroids for 2 weeks before bronchoscopy. Eight healthy nonsmoking controls and 5 non-COPD current smokers were also recruited. They had no evidence of airflow obstruction, bronchial hyperresponsiveness to hypertonic saline challenge, or chronic respiratory symptoms. Clinical examination and spirometry were performed on all individuals, whom were also questioned about the previous severity of cold symptoms. At the time of recruitment, none of the subjects had symptoms of acute respiratory tract infections for the preceding 4 weeks and did not have a diagnosis of lung cancer.

Isolation of $p B E C$ s. Human $\mathrm{pBEC}$ were obtained by endobronchial brushing during bronchoscopy in accordance with standard guidelines (54). pBECs were cultured in hormone-supplemented bronchial epithelial growth medium (BEGM, Lonza) supplemented with $50 \mathrm{U} / \mathrm{ml}$ penicillin and streptomycin as previously described (55-57). All subjects gave written informed consent.

Fbln1c targeting vector. A genomic clone approximately $14 \mathrm{~kb}$ in length containing exon 15 encoding the carboxyl terminal domain of Fbln1c was isolated from a 129/SvEvTacfBr mouse genomic library (Agilent Technologies). The clone was digested with the restriction enzymes EcoRV and XhoI to release a $6.8-\mathrm{kb}$ fragment located approximately $1.1 \mathrm{~kb}$ upstream of exon 15 (Figure 2). XhoI linkers were added to the 6.8 -kb fragment, which was subsequently cloned into a XhoI site located downstream of a HSV-tk gene to create the $5^{\prime}$ homologous long arm of the targeting vector. The $3^{\prime}$ homologous arm of the targeting vector was obtained by digestion of the genomic clone with HindIII and SpeI to release a 4.7-kb fragment located approximately $2 \mathrm{~kb}$ downstream of exon 15 . BamHI linkers were added, and the DNA was cloned into a BamHI site downstream of a loxP Neomycin (Neo) loxP resistant gene. The resulting targeting vector was linearized with NotI and transfected into ES cells by electroporation. Transfected ES cells were grown on gelatin and selected for G418 resistance. Homologous recombinant clones were identified by Southern blot analysis using probes located outside of the targeting region. Targeting of the $5^{\prime}$ arm was confirmed by the presence of a 9.8-kb fragment from the WT Fbln1 allele and a 6.8-kb fragment for the targeted Fbln 1 allele following hybridization of EcoRV-digested ES cell genomic DNA. Likewise, recombination of the 3' arm was confirmed by the presence of a 10-kb fragment derived from the WT Fbln1 allele and an 8.2-kb band from the targeted Fbln1 allele following hybridization of ApaI digested ES cell genomic DNA.

One homologous recombinant clone was expanded and injected into C57BL/ 6 blastocysts (Charles River Laboratories) and implanted into pseudopregnant females. The generated chimeric male mice were bred with 129S6/SvEvTac female mice (Taconic Biosciences), and their offspring were genotyped by PCR using the oligonucleotide primers 5'-GGCGCGAAGGGGCCACCAAAGAACGGAG-3', 5'-GTGCTAA GGTGAGAATAAGTTCTCTTAGTAGCATC-3', and 5'-GCCTGATAAGCATCCAATAAGACACAA AC-3' to identify pups carrying the targeted $F b l n 1$ allele. To excise the loxP Neo loxP cassette, male offspring carrying the targeted Fbln1 allele were mated to female ZP3-Cre mice (C57BL/6-Tg[Zp3-cre]93Knw/J, catalog 003651, The Jackson Laboratory) as previously described (58). Pups from the resulting cross were screened by PCR for the Cre-mediated recombination event using oligonucleotide primers 5'-GCCTGATAAGCATCCAATAAGACACAAAC-3', 5'-GGGCTGCAGGAATTCGATATC-3', and 5'-CAGGTTCTTACTTCCTGTGACAG-3'. Mice carrying the targeted $F b \ln 1$ allele following removal of 
the Neo cassette were backcrossed to C57BL/ 6 mice for 5 generations.

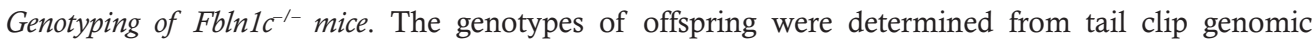
DNA by PCR using 3 oligonucleotide primers. To detect the WT Fbln 1 allele, PCR was performed using 5'-GCCTGATAAGCATCCAATAAGACACAAAC-3' and 5'-CAGGTTCTTACTTCCTGTGACAG-3'. To detect heterozygous and homozygotes mice, the former primer was used with $5^{\prime}$-GGGCTGCAGGAATTCGATATC-3'. Cycling parameters for PCR were: 39 cycles of $95^{\circ} \mathrm{C}$ for 50 seconds, $53^{\circ} \mathrm{C}$ for 30 seconds, and $72^{\circ} \mathrm{C}$ for 2 minutes. The expected size for the amplicons produced from the WT allele is 532 bp, and the expected size from the targeted Fbln $1 c$ allele is $440 \mathrm{bp}$.

Detection of Fbln1 variants. Total RNA was isolated from tissues using TRIzol (Invitrogen) and an RNeasy Mini Kit (QIAGEN). cDNA was prepared from total RNA using the iScript cDNA synthesis kit (Bio-Rad) according to the manufacturer's specifications. cDNA preparations were diluted to $25-50 \mu 1$, and $2 \mu 1$ aliquots were used in PCR reactions. Oligonucleotide primers used in PCR reactions were 5'-GCCCTCCTCATTGCCAGCGGTGATGGC-3' (Fbln1c transcript), 5'-GGAGTCTCGAAGGTTCCCTTCTGTGATG-3' (Fbln1d transcript), and 5'-CCCAATGGCCGCAACTGCCAAGACATTG-3' (common primer for $F b \ln 1 c$ and $F b \ln 1 d$ ). To detect $F b \ln 1 c$ and $F b \ln 1 d$ variants, all 3 primers were used with the following cycling parameters: 29 cycles of $95^{\circ} \mathrm{C}$ for 50 seconds, $65^{\circ} \mathrm{C}$ for 45 seconds, and $72^{\circ} \mathrm{C}$ for 1 minute. The expected size for the amplicons produced from Fbln $1 \mathrm{c}$ is $300 \mathrm{bp}$ and the expected size for Fbln $1 \mathrm{~d}$ is $380 \mathrm{bp}$.

Experimental mouse models and interventions. Six- to 8-week-old female WT or Fbln1c-/ C57BL/6J mice (Medical University of South Carolina) were exposed to twelve 3R4F cigarettes (University of Kentucky) using a custom-designed and purpose-build nose-only smoke system ( $\mathrm{CH}$ Technologies) twice per day with at least 90 minutes of rest in between, as previously described (18-22). They were exposed either for 4 days or 5 times per week for 4,6 , or 8 weeks.

Fbln1c siRNA (sense: 5'-CUGCAAAGAUAUUGACGAAUU-3', antisense: 5'-UUCGUCAAUAUCUUUGCAGUU-3'), and negative control scrambled siRNA (sense: 5'-UGGUUUACAUGUUGUGUGAUU-3', antisense: 5'-UCACACAACAUGUAAACCAUU-3') were obtained from Dharmacon. Mice were treated intranasally with $40 \mu \mathrm{g}$ of either Fbln1c or scrambled siRNA 2 hours before CS exposure every second day for 4 days or 3 times per week from week 6-8 of exposure.

Experimental chronic asthma was induced by intranasal administration of house dust mite (HDM) extract (Greer Laboratories) at $25 \mu \mathrm{g}$ in sterile saline as described previously (59). Control mice received sterile saline only. HDM was administered for 5 consecutive days per week for 5 weeks.

Experimental pulmonary fibrosis was induced by intranasal administration of one dose of bleomycin sulphate (MP Biomedical) at $0.05 \mathrm{U} /$ mouse as described previously (60). Control groups received an equal volume of sterile PBS. Tissue collection was performed 28 days after bleomycin treatment.

$B A L F$. Mouse mutilobed lungs were tied off, and BALF was collected from the single-lobed lung by washing twice with PBS (500 $\mu 1)$. Cells were pelleted ( $150 \mathrm{~g}, 10$ minutes) and resuspended in rbc lysis buffer. Remaining cells were cytocentrifuged ( $300 \mathrm{~g}, 5$ minutes, ThermoFisher Scientific) onto microscope slides. BALF slides were stained with May-Grunwald-Giemsa, and differential counts were enumerated according to morphological criteria using light microscopy as previously described (61).

Lung RNA extraction and real-time PCR. Whole lungs were excised and homogenized using a tissuetearor stick homogenizer (BioSpec). Total RNA was extracted using TRIzol (Invitrogen) according to the manufacturer's instructions (62). RNA (1,000 ng) from whole lungs was reverse transcribed using Bioscript (Bioline) and random hexamer primers (Invitrogen) (63). The mRNA expression of Col1a1, Fbln1c, Fbln1d, Smad2, Smad3, Smad4, MMP1, MMP3, MMP7, MMP8, MMP12, and MMP13 (Supplemental Table 3) was determined using real-time PCR and a Viia 7 real-time PCR system (Invitrogen) and compared with the reference gene hypoxanthine-guanine phosphoribosyltransferase (HPRT).

ECM array. RNA was extracted from mouse lung tissues and reversed transcribed to cDNA. cDNA were hybridized to an $\mathrm{RT}^{2}$ profiler Mouse Extracellular Matrix PCR array (QIAGEN) that contained 84 ECMspecific genes and housekeeping genes. PCR was performed according to the manufactures' instructions. $\mathrm{RT}^{2}$ Profiler PCR Array Data Analysis software (version 3.5, QIAGEN) was used to normalize Ct amplification values to housekeeping genes. Data were expressed as $\Delta \Delta^{\mathrm{Ct}}$, and fold-change was compared with normal air-exposed controls.

Airway remodeling. Mouse lungs were perfused with $0.9 \%$ saline and formalin fixed, and sections were cut to $4-\mu \mathrm{m}$ thickness. Slides were deparaffinized with xylene and a graded ethanol series. Collagen was stained with Verhoff's-Van Gieson (VVG) stain (Australian Biostain). Photomicrographs were taken and 
images were evaluated with Image J (version 1.47) as previously described (20, 22).

Briefly, at least 6 airways per mouse were blind-selected, and those from 4-6 animals in each experimental group were examined with a light microscope (BX41, Olympus). Morphometric parameters were marked manually on the digital representation of the airways using Image-pro plus software (version 7). Airways were divided into 3 categories according to the perimeter of their basement membrane (Pbm): $\mathrm{Pbm} \leq 1 \mathrm{~mm}$ (small), $\mathrm{Pbm} \leq 2 \mathrm{~mm}$ (medium), and $\mathrm{Pbm}>2 \mathrm{~mm}$ (large) (64).

The width of $\mathrm{Pbm}$, the inner collagen area (Ai), and the outer collagen area (Ao) were manually measured using Image J. The collagen area (Wct) was calculated (Wct = Ao - Ai) and normalized to the Pbm.

Emphysema-like alveolar enlargement. Emphysema was assessed using the mean linear intercept technique as previously described (57). Briefly, random images of H\&E-stained lung sections were captured under $\times 40$ magnification. An 11-horizontal line template was used to overlay the first 10 images that did not contain airways and/or blood vessels. Intercepts of alveolar walls with lines were enumerated and alveolar diameter calculated by dividing the total length of the 11 lines by the average number of intercepts per lung section.

Lung function. Static lung compliance was measured by quasistatic pressure-volume loops from Flexivent apparatus (Scireq) as previously described (65). Mice were anesthetized (50 $\mu 1 / 10 \mathrm{~g}$ i.p.) with a mixture of xylazine ( $2 \mathrm{mg} / \mathrm{ml}$, Troy Laboratories) and ketamine ( $40 \mathrm{mg} / \mathrm{ml}$, Ceva). Cannulae were inserted into mouse tracheas after tracheostomy. Animals were ventilated with a tidal volume of $8 \mathrm{ml} / \mathrm{kg}$ at a rate of 450 breaths/min, with increasing airway pressure from $2-30 \mathrm{cmH}_{2} \mathrm{O}$ into the lung tissue. The volume of air in the lung at the end of maximal inspiration was determined. Static lung compliance was calculated as volume change divided by applied pressure change.

Hydroxyproline. Hydroxyproline content was used to quantify collagen in whole mouse lungs and was measured colorimetrically as described previously with modifications (66). Briefly, lung tissue was excised and snap frozen at $-80^{\circ} \mathrm{C}$. Wet lungs were weighed and homogenized in $6 \mathrm{~N} \mathrm{HCl}$ at $130^{\circ} \mathrm{C}$ for 8 hours. Five $\mu \mathrm{l}$ of each sample was mixed with $5 \mu \mathrm{l}$ of citrate-acetate buffer ( $5 \%$ citric acid, $1.2 \%$ glacial acetic acid, $7.24 \%$ sodium acetate, and 3.4\% sodium hydroxide). Chloramine-T solution (100 $\mu 1,1.4 \%$ chloramine-T, $10 \%$ $\mathrm{N}$-propanol, and $80 \%$ citrate-acetate buffer) was added, and samples were incubated at room temperature for 20 minutes. Ehrlich's solution (100 $\mu$, Sigma-Aldrich) was added, and the mix was incubated at $65^{\circ} \mathrm{C}$ for 18 minutes. Absorbance was measured at $558 \mathrm{~nm}$, and concentrations were determined in comparison with standard curves generated using dilutions of pure hydroxyproline (Sigma-Aldrich).

Soluble collagen. Soluble collagen in mouse lungs was determined using a Sircol Collagen Assay kit (Biocolor) according to the manufacturer's instructions. Briefly, lungs were weighed and homogenized in pepsin (Sigma-Aldrich, $0.1 \mathrm{mg} / \mathrm{ml}$ in $0.5 \mathrm{M}$ acetic acid) for 24 hours at $4^{\circ} \mathrm{C}$. Supernatants were collected after centrifugation $(150 \mathrm{~g}, 10$ minutes). Sircol dye reagent was added with shaking for 30 minutes at room temperature and were again centrifuged. Pellets were suspended in alkali reagent from the kit. Optical density was measured at $550 \mathrm{~nm}$, and the concentrations of soluble collagen were compared with standard solutions and a standard curve provided by the manufacturers.

Protein extraction. Lung tissues were thawed and homogenized in RIPA buffer (Sigma-Aldrich) supplemented with PhosSTOP phosphatase inhibitor and complete protease inhibitor cocktails (Roche Diagnostics) as previously described $(21,22)$. Tissues were homogenized and centrifuged $(8,000 \mathrm{~g}$, 10 minutes, $4^{\circ} \mathrm{C}$ ), and proteins were collected for immunoblot or ELISA. Protein concentrations were determined using BCA protein assay kit (Pierce Biotechnology).

Immunoblotting. Proteins were subjected to SDS-PAGE using Mini-PROTEAN TGX Stain-Free gels (BioRad) and transferred to PVDF (EMD Millipore). Specific antibodies were used to detect Fbln1 (clone ab175204), Colla1 (clone ab21286), and Postn (clone ab14041) (all fromAbcam); Fn (clone F3648, Sigma-Aldrich); and Tnc (clone sc-20932), Vcan (clone sc-25831), Hapln1 (clone sc-135184), and ECM1 (clone sc-135032) (all from Santa Cruz Biotechnology). $\beta$-Actin (clone ab70165) and GAPDH (clone ab9483) (both from Abcam) were used as loading controls. Images of immunoblots were captured with a ChemiDoc MP System (Bio-Rad). Some blots were cut based on the protein molecular weight. Thus, multiple proteins were detected at the same time. Some blots were stripped but only reprobed once in order to avoid background effects.

For lung and cell lysates samples, Image J was used for densitometry analysis as described previously (57). In brief, the densitometric values of proteins of interest were measured and normalized to the density of the internal control proteins, such as $\beta$-actin or GAPDH. Values were represented as fold change of the experimental compared with control groups.

For serum samples, a stain-free technology was employed for densitometry analysis using a ChemiDoc 
MP System (Bio-Rad) as described previously (67). After electrophoresis, Mini-PROTEAN TGX Stain-Free gels were activated by ultraviolet light for visualization of protein bands. The gels were then transferred to PVDF membranes, which were also visualized to capture stain-free images (ChemiDoc MP System; BioRad). For densitometry, all lanes including the molecular weight marker lanes as references were selected in the stain-free blot. A normalization factor was calculated by dividing the total intensity of the stain-free reference lane by the whole intensity of each lane. For Fbln1 immunoblots, the intensity of the protein in each lane was measured and normalized with the normalization factor for each sample. The fold change of normalized volume in each treatment group was compared with control groups.

ELISA. TNF- $\alpha$, IL-33, and CXCL1 (Duoset, R\&D Systems) levels in lung tissues and BALF supernatants were assessed by ELISA according to the manufacturers' instructions. TGF- $\beta$ levels were determined using capture and detection antibodies (BD Pharmingen) according to manufacturer's instructions. The target proteins in lungs were normalized to total lung protein.

Immunostaining. Lungs were perfused, inflated, formalin-fixed, paraffin-embedded, and sectioned (4-6 $\mu \mathrm{m})$. Longitudinal sections were deparaffinized, incubated with citrate buffer for antigen retrieval, and blocked with casein (Sigma-Aldrich, room temperature, 1 hour). Slides were incubated with Fbln1 (1:40), Tnc (1:20), Postn $(1: 1,000)$, Colla1 $(1: 200)$, and Fn $(1: 200)\left(4^{\circ} \mathrm{C}\right.$, overnight) followed by anti-rabbit horseradish peroxidaseconjugated secondary antibody (R\&D Systems, $37^{\circ} \mathrm{C}, 30$ minutes). Diaminobenzidine (DAB, DAKO) was applied on slides, and hematoxylin was used as a counterstain. $\alpha$-SMA-positive cells were stained with antiactin $\alpha$-SM-Cy3 (1:200, clone c6198, Sigma-Aldrich), and nuclei were stained with Hoechst (Sigma-Aldrich).

Some mouse lungs were perfused and inflated with OCT medium and PBS at a 1:1 ratio. Tissues were immediately placed in OCT medium and rapidly frozen on dry ice. Frozen sections $(5-\mu \mathrm{m})$ were cut and fixed in cold acetone for 10 minutes. After 2 hours of blocking with 5\% BSA (Sigma-Aldrich), slides were incubated with Col1a1, Tnc, Postn, Fbln1, and Fn antibodies with PE/Cy5 (clone ab102893), AMCA (clone ab102846), and FITC (clone ab102884) conjugation kits (all from Abcam) in different groups $\left(4^{\circ} \mathrm{C}\right.$, overnight). Fluorescence signals were examined using confocal microscopy (Nikon C2), and all images were analyzed using NIS-Element software (Nikon).

Statistics. Results are presented as mean \pm SEM from 4-8 sample size, each in duplicate or triplicate experiments. Comparisons between 2 groups were determined using unpaired 2-tailed Student's $t$ test, and multiple groups were performed by one-way ANOVA with Bonferroni post-test using Prism-GraphPad Software version 6 (GraphPad). Representative photomicrographs are shown throughout. Results are mean \pm SEM, where $P$ less than 0.05 is considered significant.

Study approval. Human studies were approved by the Human Research Ethics Committee of the University of Newcastle. All mouse experiments were approved by the animal ethics committee of the University of Newcastle.

\section{Author contributions}

GL, AGJ, JKB, and PMH participated in the design of the study. GL performed all in vivo and part of the in vitro experiments. WSA and MAC generated $F b \ln 1 c^{-1-}$ mice. PMN, TJH, MF, and SLG assisted with mouse experiments. RYK and JCH assisted with lung function experiments and emphysema analysis. MDI assisted in analyzing lung function data. GT performed parts of tissue sectioning. PABW performed subject recruitment and research bronchoscopy. ACYH and BGO performed all pBEC culturing and parts of in vitro experiments. All authors participated in the interpretation of data and preparation and editing of the manuscript for intellectual content. All authors read and approved the final manuscript (with the exception of WSA, who passed away before the final version was completed). DAK assisted with experimental design. MMW assisted with airway remodeling analysis.

\section{Acknowledgments}

This work is dedicated to the memory of W. Scott Argraves, who passed away during completion of this study. This work was supported by grants from National Health and Medical Research Council (NHMRC) of Australia to P.M. Hansbro, NIH (R01HL095067) to W.S. Argraves, and NIH (1R03DE02509) to M.C. Cooley. A.G. Jarnicki was supported by a Lung Foundation of Australia/Boerhinger Ingelheim COPD research Fellowship. B.G. Oliver and J.K. Burgess were supported by NHMRC Career Development Fellowships. P.M. Hansbro was supported by an NHMRC Principal Research Fellowship and Brawn Fellowship, Faculty of Health, University of Newcastle. This study used the services of the Gene Function Core 
at the Medical University of South Carolina, which is supported by NIH-NIGMS P20RR016434 and the Office of the Vice President for Research at the Medical University of South Carolina. We thank Kristy Wheeldon for technical assistance.

Address correspondence to: Philip M. Hansbro, Hunter Medical Research Institute and The University of Newcastle, Lot 1 Kookaburra Circuit, New Lambton Heights, New South Wales, 2305, Australia. Phone: 61.2.4042.0187; Fax 61.2.4042.0024; E-mail Philip.Hansbro@newcastle.edu.au.

1. Kessenbrock K, Plaks V, Werb Z. Matrix metalloproteinases: regulators of the tumor microenvironment. Cell. 2010;141(1):52-67.

2. Keely S, Talley NJ, Hansbro PM. Pulmonary-intestinal cross-talk in mucosal inflammatory disease. Mucosal Immunol. 2012;5(1):7-18

3. Fricker M, Deane A, Hansbro PM. Animal models of chronic obstructive pulmonary disease. Expert Opin Drug Discov. 2014;9(6):629-645.

4. Tuder RM, Petrache I. Pathogenesis of chronic obstructive pulmonary disease. J Clin Invest. 2012;122(8):2749-2755.

5. Hallstrand TS, Hackett TL, Altemeier WA, Matute-Bello G, Hansbro PM, Knight DA. Airway epithelial regulation of pulmonary immune homeostasis and inflammation. Clin Immunol. 2014;151(1):1-15.

6. Pini L, Pinelli V, Modina D, Bezzi M, Tiberio L, Tantucci C. Central airways remodeling in COPD patients. Int J Chron Obstruct Pulmon Dis. 2014;9:927-932.

7. Dolhnikoff M, et al. The outer wall of small airways is a major site of remodeling in fatal asthma. J Allergy Clin Immunol. 2009;123(5):1090-7, 1097.e1.

8. Ponticos M, et al. Pivotal role of connective tissue growth factor in lung fibrosis: MAPK-dependent transcriptional activation of type I collagen. Arthritis Rheum. 2009;60(7):2142-2155.

9. Argraves WS, Tran H, Burgess WH, Dickerson K. Fibulin is an extracellular matrix and plasma glycoprotein with repeated domain structure. J Cell Biol. 1990;111(6 Pt 2):3155-3164.

10. Tran H, VanDusen WJ, Argraves WS. The self-association and fibronectin-binding sites of fibulin-1 map to calcium-binding epidermal growth factor-like domains. J Biol Chem. 1997;272(36):22600-22606.

11. Fujiwara H, et al. The basement membrane of hair follicle stem cells is a muscle cell niche. Cell. 2011;144(4):577-589.

12. Roark EF, Keene DR, Haudenschild CC, Godyna S, Little CD, Argraves WS. The association of human fibulin-1 with elastic fibers: an immunohistological, ultrastructural, and RNA study. J Histochem Cytochem. 1995;43(4):401-411.

13. Lau JY, et al. Fibulin-1 is increased in asthma--a novel mediator of airway remodeling?. PLoS ONE. 2010;5(10):e13360.

14. Ge Q, et al. Fibulin1C peptide induces cell attachment and extracellular matrix deposition in lung fibroblasts. Sci Rep. 2015;5:9496.

15. Jaffar J, et al. Fibulin-1 predicts disease progression in patients with idiopathic pulmonary fibrosis. Chest. 2014;146(4):1055-1063.

16. Akhurst RJ, Hata A. Targeting the TGF $\beta$ signalling pathway in disease. Nat Rev Drug Discov. 2012;11(10):790-811.

17. Chen L, Ge Q, Black JL, Deng L, Burgess JK, Oliver BG. Differential regulation of extracellular matrix and soluble fibulin-1 levels by TGF- $\beta_{1}$ in airway smooth muscle cells. PLoS ONE. 2013;8(6):e65544.

18. Beckett EL, et al. A new short-term mouse model of chronic obstructive pulmonary disease identifies a role for mast cell tryptase in pathogenesis. J Allergy Clin Immunol. 2013;131(3):752-762.

19. Franklin BS, et al. The adaptor ASC has extracellular and 'prionoid' activities that propagate inflammation. Nat Immunol. 2014;15(8):727-737.

20. Hansbro PM, et al. Importance of mast cell Prss31/transmembrane tryptase/tryptase- $\gamma$ in lung function and experimental chronic obstructive pulmonary disease and colitis. J Biol Chem. 2014;289(26):18214-18227.

21. Tay HL, et al. Antagonism of miR-328 increases the antimicrobial function of macrophages and neutrophils and rapid clearance of non-typeable Haemophilus influenzae (NTHi) from infected lung. PLoS Pathog. 2015;11(4):e1004549.

22.Haw TJ, et al. A pathogenic role for tumor necrosis factor-related apoptosis-inducing ligand in chronic obstructive pulmonary disease [published online ahead of print November 11, 2015]. Mucosal Immunol. doi: 10.1038/mi.2015.111.

23. Kostka G, et al. Perinatal lethality and endothelial cell abnormalities in several vessel compartments of fibulin-1-deficient mice. Mol Cell Biol. 2001;21(20):7025-7034.

24. Cooley MA, et al. Fibulin-1 is required for morphogenesis of neural crest-derived structures. Dev Biol. 2008;319(2):336-345.

25. Zafra MP, et al. Gene silencing of SOCS3 by siRNA intranasal delivery inhibits asthma phenotype in mice. PLoS ONE. 2014;9(3):e91996.

26. Sorokin L. The impact of the extracellular matrix on inflammation. Nat Rev Immunol. 2010;10(10):712-723.

27. Kearley J, et al. Cigarette smoke silences innate lymphoid cell function and facilitates an exacerbated type I interleukin-33-dependent response to infection. Immunity. 2015;42(3):566-579.

28. Zandvoort A, Postma DS, Jonker MR, Noordhoek JA, Vos JT, Timens W. Smad gene expression in pulmonary fibroblasts: indications for defective ECM repair in COPD. Respir Res. 2008;9:83.

29. Zandvoort A, et al. Altered expression of the Smad signalling pathway: implications for COPD pathogenesis. Eur Respir J. 2006;28(3):533-541.

30. Chen $\mathrm{H}$, et al. Abnormal mouse lung alveolarization caused by Smad3 deficiency is a developmental antecedent of centrilobular emphysema. Am J Physiol Lung Cell Mol Physiol. 2005;288(4):L683-L691.

31. Sasaki T, Mann K, Murphy G, Chu ML, Timpl R. Different susceptibilities of fibulin-1 and fibulin-2 to cleavage by matrix metalloproteinases and other tissue proteases. Eur J Biochem. 1996;240(2):427-434.

32. Wang $Q$, et al. Extracellular calumenin suppresses ERK1/2 signaling and cell migration by protecting fibulin-1 from MMP13-mediated proteolysis. Oncogene. 2015;34(8):1006-1018. 
33. Scheibner KA, Lutz MA, Boodoo S, Fenton MJ, Powell JD, Horton MR. Hyaluronan fragments act as an endogenous danger signal by engaging TLR2. J Immunol. 2006;177(2):1272-1281.

34. Pierce JA, Hocott JB. Studies on the collagen and elastin content of the human lung. J Clin Invest. 1960;39:8-14.

35. Hogg JC, et al. Micro-computed tomography measurements of peripheral lung pathology in chronic obstructive pulmonary disease. Proc Am Thorac Soc. 2009;6(6):546-549.

36. Churg A, Zhou S, Preobrazhenska O, Tai H, Wang R, Wright JL. Expression of profibrotic mediators in small airways versus parenchyma after cigarette smoke exposure. Am J Respir Cell Mol Biol. 2009;40(3):268-276.

37. Wu YJ, La Pierre DP, Wu J, Yee AJ, Yang BB. The interaction of versican with its binding partners. Cell Res. 2005;15(7):483-494.

38. Balbona K, Tran H, Godyna S, Ingham KC, Strickland DK, Argraves WS. Fibulin binds to itself and to the carboxyl-terminal heparin-binding region of fibronectin. J Biol Chem. 1992;267(28):20120-20125.

39. Twal WO, et al. Fibulin-1 suppression of fibronectin-regulated cell adhesion and motility. J Cell Sci. 2001;114(Pt 24):4587-4598.

40. Williams SA, Schwarzbauer JE. A shared mechanism of adhesion modulation for tenascin-C and fibulin-1. Mol Biol Cell. 2009;20(4):1141-1149

41. Norris RA, et al. Periostin regulates collagen fibrillogenesis and the biomechanical properties of connective tissues. J Cell Biochem. 2007;101(3):695-711.

42. Engvall E, Ruoslahti E. Binding of soluble form of fibroblast surface protein, fibronectin, to collagen. Int J Cancer. 1977;20(1):1-5.

43. Naba A, Clauser KR, Hoersch S, Liu H, Carr SA, Hynes RO. The matrisome: in silico definition and in vivo characterization by proteomics of normal and tumor extracellular matrices. Mol Cell Proteomics. 2012;11(4):M111.014647.

44. Wells JM, Gaggar A, Blalock JE. MMP generated matrikines. Matrix Biol. 2015;44-46:122-129.

45. Grainge CL, et al. Effect of bronchoconstriction on airway remodeling in asthma. N Engl J Med. 2011;364(21):2006-2015.

46. Kurowska-Stolarska M, et al. IL-33 amplifies the polarization of alternatively activated macrophages that contribute to airway inflammation. J Immunol. 2009;183(10):6469-6477.

47. Saglani S, et al. IL-33 promotes airway remodeling in pediatric patients with severe steroid-resistant asthma. J Allergy Clin Immunol. 2013;132(3):676-685.e13.

48. Carey WA, Taylor GD, Dean WB, Bristow JD. Tenascin-C deficiency attenuates TGF-ß-mediated fibrosis following murine lung injury. Am J Physiol Lung Cell Mol Physiol. 2010;299(6):L785-L793.

49. Bentley JK, et al. Periostin is required for maximal airways inflammation and hyperresponsiveness in mice. J Allergy Clin Immunol. 2014;134(6):1433-1442.

50. Kusubata M, et al. Spatiotemporal changes of fibronectin, tenascin-C, fibulin-1, and fibulin-2 in the skin during the development of chronic contact dermatitis. J Invest Dermatol. 1999;113(6):906-912.

51. Midwood K, et al. Tenascin-C is an endogenous activator of Toll-like receptor 4 that is essential for maintaining inflammation in arthritic joint disease. Nat Med. 2009;15(7):774-780.

52. Timpl R, Sasaki T, Kostka G, Chu ML. Fibulins: a versatile family of extracellular matrix proteins. Nat Rev Mol Cell Biol. 2003;4(6):479-489.

53. Pauwels RA, Buist AS, Calverley PM, Jenkins CR, Hurd SS. Global strategy for the diagnosis, management, and prevention of chronic obstructive pulmonary disease. NHLBI/WHO Global Initiative for Chronic Obstructive Lung Disease (GOLD) Workshop summary. Am J Respir Crit Care Med. 2001;163(5):1256-1276.

54. Workshop summary guidelines: investigative use of bronchoscopy, lavage, bronchial biopsies in asthma other airway diseases J Allergy Clin Immunol. 1991;88(5):808-814.

55. Hsu AC, Barr I, Hansbro PM, Wark PA. Human influenza is more effective than avian influenza at antiviral suppression in airway cells. Am J Respir Cell Mol Biol. 2011;44(6):906-913.

56. Parsons KS, Hsu AC, Wark PA. TLR3 and MDA5 signalling, although not expression, is impaired in asthmatic epithelial cells in response to rhinovirus infection. Clin Exp Allergy. 2014;44(1):91-101.

57. Hsu AC, et al. Targeting PI3K-p110 $\alpha$ suppresses influenza virus infection in chronic obstructive pulmonary disease. Am J Respir Crit Care Med. 2015;191(9):1012-1023.

58. van Palenstein Helderman WH. Lysozyme concentrations in the gingival crevice and at other oral sites in human subjects with and without gingivitis. Arch Oral Biol. 1976;21(4):251-255.

59. Fattouh R, et al. Transforming growth factor-beta regulates house dust mite-induced allergic airway inflammation but not airway remodeling. Am J Respir Crit Care Med. 2008;177(6):593-603.

60. Gold MJ, et al. Mucosal production of uric acid by airway epithelial cells contributes to particulate matter-induced allergic sensitization. Mucosal Immunol. 2016;9(3):809-820.

61. Preston JA, et al. Inhibition of allergic airways disease by immunomodulatory therapy with whole killed Streptococcus pneumoniae. Vaccine. 2007;25(48):8154-8162.

62. Collison A, et al. The E3 ubiquitin ligase midline 1 promotes allergen and rhinovirus-induced asthma by inhibiting protein phosphatase 2A activity. Nat Med. 2013;19(2):232-237.

63. Horvat JC, et al. Early-life chlamydial lung infection enhances allergic airways disease through age-dependent differences in immunopathology. J Allergy Clin Immunol. 2010;125(3):617-25, 625.e1-625.e6.

64. Palmans E, Kips JC, Pauwels RA. Prolonged allergen exposure induces structural airway changes in sensitized rats. Am J Respir Crit Care Med. 2000;161(2 Pt 1):627-635.

65. Harris RS. Pressure-volume curves of the respiratory system. Respir Care. 2005;50(1):78-98.

66. Woessner JF. The determination of hydroxyproline in tissue and protein samples containing small proportions of this imino acid. Arch Biochem Biophys. 1961;93:440-447.

67. Gürtler A, et al. Stain-Free technology as a normalization tool in Western blot analysis. Anal Biochem. 2013;433(2):105-111. 